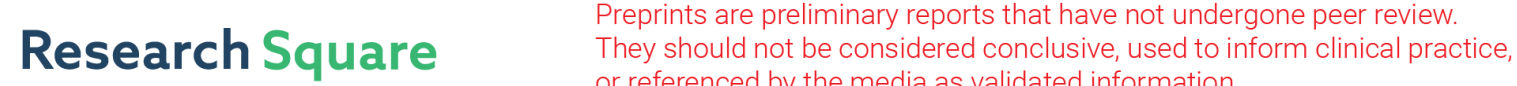 \\ or referenced by the media as validated information. \\ Simulation and Experimental Research on the Liquid Spreading Behavior in the Wire Sawn Kerf
}

\section{Lin Lin}

Huaqiao University https://orcid.org/0000-0002-5132-5142

Hui Huang ( $\nabla$ huanghuihh@hotmail.com )

Huaqiao University https://orcid.org/0000-0001-6690-8984

\section{Original Article}

Keywords: Liquid, Spreading regime, Kerf, CFD, non-dimensional analysis, Prediction model

Posted Date: April 9th, 2021

DOI: https://doi.org/10.21203/rs.3.rs-322923/v1

License: (c) (i) This work is licensed under a Creative Commons Attribution 4.0 International License. Read Full License 


\section{Title page}

\section{Simulation and Experimental Research on the Liquid Spreading Behavior in the Wire Sawn Kerf}

Lin Lin, born in 1993, is currently a master of engineering. He received his master degree from Huaqiao University, China, in 2020. Tel: +86-17359899069; E-mail: linlin.rylynn@gmail.com

Hui Huang, born in 1974, is currently a professor at Huaqiao University, China. He received his Ph. D degree from Nanjing University of Aeronautics and Astronautics, China, in 2002. His research interests include the machining of brittle material and the manufacturing of superabrasive tools.

Tel: +86-592-6162615; E-mail: huanghuihh@hotmail.com

Corresponding author: Hui Huang E-mail: huanghuihh@hotmail.com 


\title{
Simulation and Experimental Research on the Liquid Spreading in the Wire Sawn Kerf
}

\author{
Lin Lin ${ }^{1}$ Hui Huang ${ }^{2}$
}

Received March xx, 202x; revised March xx, 202x; accepted March xx, 202x

(C) Chinese Mechanical Engineering Society and Springer-Verlag Berlin Heidelberg 2017

\begin{abstract}
The significance of liquid for abrasive wire sawing has been demonstrated by researchers with considerable studies. However, its performance in the spreading behavior is limited by the development trend of larger area wafer and narrower slicing kerf. Nevertheless, studies on the liquid spreading behavior in the wire sawing kerf are awfully limited. In this paper, a 3D CFD (Computational Fluid Dynamics) model was presented to simulate the liquid spreading behavior in the kerf. Where a VOF (Volume of Fluid) method with a CSF (Continuum Surface Force) model is used to simulate multiphase flow, and an empirical correlation for characterizing the liquid dynamic contact angle is introduced using UDF (User Defined Functions). Parametric simulations were performed on the kerf area, kerf width, liquid viscosity, liquid surface tension and liquid velocity at the inlet area of the kerf. Verification experiments are conducted for the validity of the simulation model. From both simulation and experimental results, three typical liquid spreading regimes in the kerfs are found, which perform distinct different effects on wire sawing. Moreover, the limiting conditions of the three spreading regimes are identified by non-dimensional analysis, then a prediction model is proposed for the liquid spreading regime, by given a set of Weber number and Capillary number. For wire sawing, the increase in the wafer area will not change the liquid spreading regime in the kerf, but the reduction of the kerf width will significantly hinder the liquid spreading behavior. By adjusting the physical properties and supply conditions of the liquid, the spreading regime can be effectively converted to facilitate wire sawing.

$\triangle$ Hui Huang

huanghuihh@hotmail.com

1 State Key Institute of Manufacturing Engineering, Huaqiao University, Xiamen, 361021, Fujian Province, P. R. China

2 State Key Institute of Manufacturing Engineering, Huaqiao University, Xiamen, 361021, Fujian Province, P. R. China
\end{abstract}

Keywords: Liquid - Spreading regime - Kerf - CFD • non-dimensional analysis $\bullet$ Prediction model

\section{Introduction}

Abrasive wire sawing technology has been widely applied in semiconductor and photovoltaic industry for wafer production. Since 1990s, multi-wire slurry sawing (MWSS) was applied to slicing silicon ingot, due to its high throughput, smaller kerf loss, and the ability to slice large-size ingots [1]. Recently, fixed diamond wire sawing (DWS) is promoted and has achieved its industrial status, owing to its efficient material removal [2]. In abrasive wire sawing, cutting liquid is sprayed directly into the kerf or to the high-speed moving $(5-30 \mathrm{~m} / \mathrm{s})$ wire web then to be carried into the sawing channel, as shown in Figs. 1(a)-(b). The significance of liquid should not be underestimated due to its main functions of lubrication, cooling, and chip removal [3]. Especially for MWSS, cutting fluid, as a carrier for abrasive particles, brings abrasive into the sawing channel to achieve material removal.

Considerable research efforts have been devoted to the liquid behavior in abrasive wire sawing on account of its indispensable role. So far, most studies have focused on MWSS because of its earlier development. The early work was performed when Bhagavat and Kao [4] proposed a 2D model of elasto-hydrodynamic (EHD) interaction between wire and slurry. On the assumption of incompressible, steady and laminar flow, the finite element method was used to analyze the slurry film thickness and pressure distribution. Möller et al. [1] also presented a 2D model to analyze the EHD behavior of slurry. Extensive studies have dealt with the abrasive behavior in the slurry by Bhagavat et al [4] and Yang et al [5], which clarify the prominent 


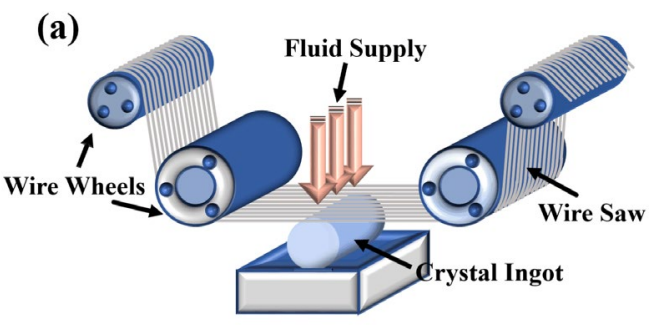

(b)

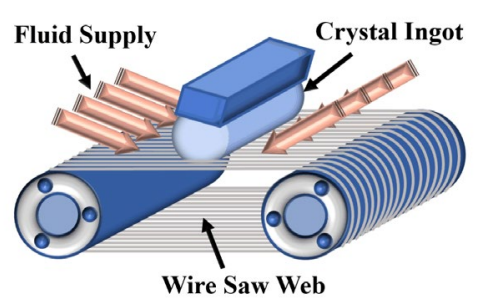

Figure 1 Two main liquid-supply modes in abrasive wire sawing: (a) spray into the kerf, (b) spray to the moving wire web.

impact of liquid film thickness and pressure distribution on material removal. Furthermore, the significance of coolant liquid on the thermal environment of wire sawing was demonstrated by Kao et al [6] and Johnson et al [7] by finite element simulation.

Although it is not clearly stated, in all the above studies, it is acquiesced that the liquid can enter the sawing channel and remain in a continuous and saturated state. However, a crucial question was overlooked: whether and how the liquid could enter the sawing area. Since the formation of the air layer in the sawing channel was observed by Ishikawa et al. [8], and Nassauer et al. [9] also observed the micro bubbles emerging and collapsing phenomenon in the sawing experiments. It is confirmed that there is air in the sawing channel, wherein the liquid is not saturated, which may prevent the liquid from performing its function, and even dry cutting may occur.

We have witnessed in recent years the development of abrasive wire sawing technology, using the many advances in fabrication to give larger wafer diameters (from 2 inches to 12 inches) as well as smaller wire diameters for less kerf loss (the current minimum wire diameter is up to $60 \mu \mathrm{m}$ ). In this trend, there appears larger sawing kerf area, narrower kerf width in the sawing process. The complex fluid environment at small scale creates entirely new challenges to the entering-spreading behavior of the liquid in the kerf. Nevertheless, for abrasive wire sawing, neither the entering-spreading behavior of the liquid nor the introduction of air is explicit represented, and the liquid-supply mode of directly spraying on the kerf has not been investigated. Moreover, most of the above studies use 2D models for simulation, nevertheless, 2D model has inevitable limitations for the simulation of actual conditions.

In this paper, a 3D CFD model is constructed to simulate the liquid spreading behavior in the kerf. Parametric simulations are performed on the kerf area, kerf width, liquid viscosity, liquid surface tension and the liquid velocity at the inlet area of the kerf. Further, the validity of the simulation model is verified by experiments.

\section{Simulation and experimental methodology}

This paper aims at investigating the liquid spreading behavior in the kerf, focusing on the liquid supply mode of directly sprayed in the kerf. To simplify the model, single wire sawing of sapphire ingot is taken as an example.

\subsection{The simulation model and boundary condition}

(a)

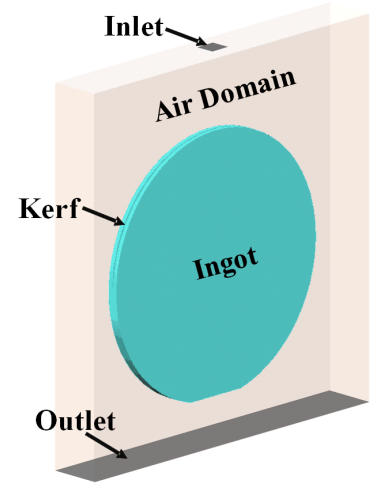

(c)

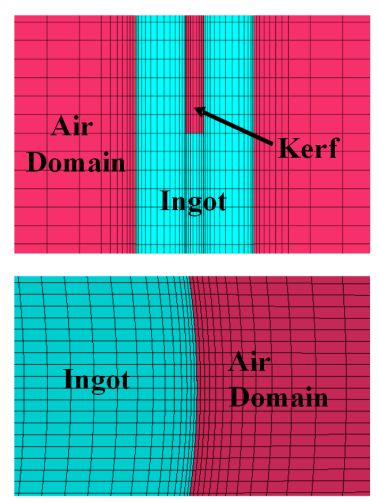

(b)

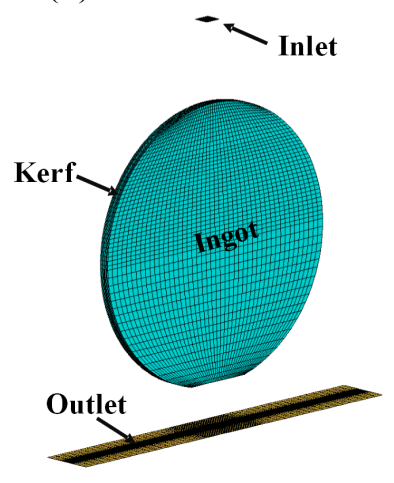

(d)

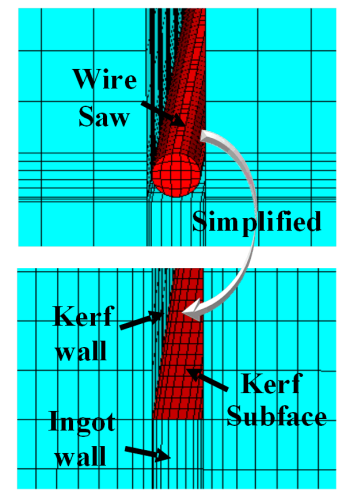

Figure 2 Geometric model and grids: (a) the calculation domain and geometric model, (b) the grid of the ingot with a kerf, (c) the local mesh densification at the wall of ingot and the kerf, (d) the simplification of the sawing area.

For the physical phenomenon of interest, a series of 3D geometry models in different dimensions are built for parametric simulation (the geometric dimensions of the models are shown in Appendix A), and the structural 
features of the models are shown in Fig. 2. As shown in Fig. 2(a), the calculation domain is a cuboid, and the inlet, a square orifice of $4 \times 4 \mathrm{~mm}$, is set at the center of the its top. In the calculation domain, an ingot model, $15 \mathrm{~mm}$ below the orifice, is built referring to the nozzles position in general sawing. To reduce computational load, the ingot model was simplified to a thickness of 2 millimeters, and a kerf was processed at the central section of the ingot (Fig. 2(a)). Moreover, as presented in Fig. 2(d), the sawing area at the kerf subface is simplified as a flat without sawing wire.

The geometric models are meshed to structured hexahedral grids, as shown in Fig. 2(b). Moreover, the local mesh densification is carried out for the wall of ingot and the kerf, as shown in Fig. 2(c). Furthermore, the grid size independence is made to enhance the accuracy and efficiency of the calculation.

The boundary condition of simulation shown in Table 1 . All walls are assumed to be uniform sand-grain surfaces with certain roughness heights $K_{s}$ (the determination of the $K_{s}$ depends on the measured surface roughness). In addition, the operating pressure is $101325 \mathrm{~Pa}$ and the gravitational acceleration $g$ is $9.8 \mathrm{~m} / \mathrm{s}^{2} . \quad v_{0}$ is the inlet velocity.

Table 1 Boundary conditions

\begin{tabular}{cc}
\hline Zone & Setting \\
\hline Inlet & Area: $9 \mathrm{~mm}^{2}$, Velocity-inlet: $v_{0}=0.1 \sim 1.6 \mathrm{~m} / \mathrm{s}$ \\
Outlet & Pressure-outlet, Gauge pressure: 0 \\
Ingot outer wall & $K_{s}=0.001 \mathrm{~mm}$ \\
Inner kerf wall & $K_{s}=0.005 \mathrm{~mm}$ \\
Kerf subface & $K_{s}=0.030 \mathrm{~mm}$
\end{tabular}

\subsection{Experimental setup}

The experimental setup (see Fig. 3) consists of the following parts: (a) pump, (b) precision valve, (c) float flowmeter, (d) 3D printing nozzle (with a $4 \times 4 \mathrm{~mm}$ square outlet), (e) liquid recovery tank, (f) three-axis linkage platform, (g) high-speed camera (Phantom/V2511). Where parts (a)-(d) are connected by water pipes and work on the liquid recovery tank for flow controlling and recovery. Meanwhile, the three-axis linkage platform is fixed at the bottom of the tank, then a 2-inch ingot with a sawn kerf is fixed on the platform (the sawing parameters are shown in appendix B). Further, in order to compare the experimental and simulation models accurately, the position of the ingot is adjusted by a three-axis linkage platform, so that the ingot is set $15 \mathrm{~mm}$ below the nozzle. Thus, after liquid flows out from the nozzle at a certain flow rate, the entire process of liquid entering and spreading in the kerf can be captured by the high-speed camera.

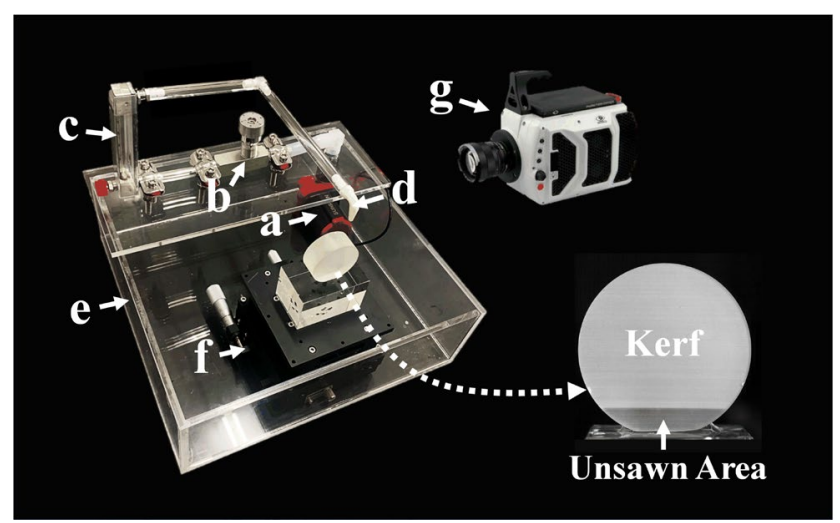

Figure 3 Experimental setup.

To clarify the effect of liquid physical properties, three solutions were prepared for simulation and experimental studies, as show in Table 2. Where $\mu_{l}, \rho_{l}$ and $\gamma_{l v}$ are the liquid dynamic viscosity, liquid density and liquid surface tension, respectively. The solution preparation refers to the cutting fluid commonly used in DWS (mainly water-based coolant) and MWSS (mainly composed of polyethylene glycol (PEG)) [6], respectively.

Table 2 Physical properties of different solutions (where $\mathrm{L}$ is a commercially available cutting fluid, solution $\mathrm{A}$ is the $2 \% \mathrm{~L}$ aqueous solution, solution B is the PEG 200 solution, solution C is the deionized water. All the solutions are prepared and measured at room temperature of $298.15 \mathrm{k}$ ).

\begin{tabular}{cccc}
\hline Solution & $\mu_{l}(\mathrm{mPa} \cdot \mathrm{s})$ & $\rho_{l}\left(\mathrm{~kg} / \mathrm{m}^{3}\right)$ & $\gamma_{l v}(\mathrm{mN} / \mathrm{m})$ \\
\hline $\mathrm{A}$ & 1.53 & 999.12 & 49.10 \\
B & 31.65 & 1122.37 & 45.71 \\
$\mathrm{C}$ & 0.92 & 997.31 & 71.92 \\
\hline
\end{tabular}

\subsection{Simulation parameters}

The basic parameters influencing the liquid spreading process typically include the liquid properties, the flow velocity, and the scale parameters of the flow environment etc. [10]. Therefore, simulations parameters is chosen as follow: (1) $A_{k}$ : area of the kerf center section, (2) $D_{w}$ : kerf width, (3) $\mu_{l}$ : liquid dynamic viscosity, (4) $\gamma_{l v}$ : liquid surface tension, (5) $v_{i}$ : liquid velocity at the inlet area of the kerf. Assume that the liquid density $\rho_{l}=$ 999.12 is a fixed value in the simulation study.

Variations of simulation parameters are shown in Table 3. $A_{k}$ and $D_{w}$ are set referring to the development trend in the industry (ingot diameter $D_{i}=2,6,12$ inch) and the actual processing conditions (sawing depth $D_{s}=D_{i} / 5$, 
$\left.D_{i} / 2,4 D_{i} / 5\right)$. Meanwhile, the liquid properties are set within a reasonable range, which refers to the physical properties of the solutions (see Table 2). According to Bernoulli's theorem [11], variations in $v_{i}$ are determined by the inlet velocity $v_{0}$ (see Table 1 ) and the distance $h_{i}$ between the inlet and the top of kerf:

$v_{i}=\sqrt{v_{0}^{2}+2 g h_{i}}=\sqrt{v_{0}^{2}+2 \times 9.8 \times 0.015}=\sqrt{v_{0}^{2}+0.294}(1)$

Table 3 Variations of simulation parameters

\begin{tabular}{cc}
\hline Parameters & Variations \\
\hline$A_{k}$ & $223.14,1013.40,1782.94,9120.73,35342.91 \mathrm{~mm}^{2}$ \\
$D_{w}$ & $0.1,0.15,0.2,0.25,0.3,0.4,0.5 \mathrm{~mm}$ \\
$\mu_{l}$ & $0.92 \sim 32.61 \mathrm{mPa} \cdot \mathrm{s}$ \\
$\gamma_{l v}$ & $20 \sim 80 \mathrm{mN} / \mathrm{m}$ \\
$v_{i}$ & $0.55,0.58,0.67,0.97,1.68 \mathrm{~m} / \mathrm{s}$ \\
\hline
\end{tabular}

Moreover, three non-dimensional numbers: Reynolds number $R e=\rho_{l} v l_{0} / \mu_{l}$, Weber number $W e=\rho_{l} v^{2} l_{0} / \gamma_{l v}$ and Capillary number $C a=v \mu_{l} / \gamma_{l v}$ are introduced to characterize liquid flow state and analyze simulation results [12]. Where $v$ is the flow velocity of the fluid; $l_{0}$ is the characteristic length. For the liquid flow in the kerf, similar to the flow between parallel plates, $l_{0}$ is seen equal to the kerf width $D_{w}$ [13]. In this study, the maximum Reynolds number $R e_{\max }$ in the kerf can be given approximately:

$R e_{\max } \approx \frac{\rho_{l \max } v_{i \max } l_{0 \max }}{\mu_{l \min }}=\frac{1124.1 \times 1.68 \times 0.0005}{0.00092}=1026.35$

Since $R e_{\max }$ is less than the lower bound of the critical Reynolds number $R e_{\mathrm{c}}$ of 2000 [13], the liquid in the kerf is laminar. For $R e \gg 1$, the quantity of interest for the effect of surface tension is determined by the Weber number [14], and the minimum weber number is approximated as follow:

$W e_{\min } \approx \frac{\rho_{l \min } v_{i \min }{ }^{2} l_{0 \min }}{\gamma_{l v \min }}=\frac{999.12 \times 0.55^{2} \times 0.0001}{0.08}=0.37$

Hence, the effect of surface tension should not be neglected since the Weber number $W e<1[10]$.

\subsection{Governing equations and solving method}

Since two-phase flow is involved in this study, a VOF (Volume of Fluid) method was introduced to capture the gas-liquid interface. In the VOF model, the volume fraction of each fluid is tracked down every cell throughout the domain [15]. For the fluid $i$, the volume fraction $\alpha_{i}$ is defined as follows:

$\alpha_{i}=0:$ The cell is empty of the fluid $i$.

$\alpha_{i}=1$ : The cell is full of the fluid $i$.

$\alpha_{i}<0$ : The cell has an interface between the fluid $i$ and one or more other fluids.

A continuity equation for the volume fraction is solved to track the interface. If the subscript $i$ represents the fluid $i$, then the continuity equation has the following form [15]:

$$
\frac{\partial \alpha_{i}}{\partial t}+\nabla \cdot\left(\alpha_{i} \vec{v}_{i}\right)=0
$$

Moreover, the fluid properties in the continuity equation are determined by their phase components in each control volume. For the two-phase flow in this study, the fluid properties of density and viscosity is given by:

$$
\begin{aligned}
& \rho=\alpha_{j} \rho_{j}+\alpha_{i} \rho_{i} \\
& \mu=\alpha_{j} \mu_{j}+\alpha_{i} \mu_{i}
\end{aligned}
$$

Where the subscript $j$ represents the fluid $j$. Depending on the fluid properties, a momentum equation is solved for the velocity field which is shared among the phases [15]. It is shown below

$\frac{\partial}{\partial t}(\rho \vec{v})+\nabla \cdot(\rho \vec{v} \vec{v})=-\nabla p+\nabla \cdot\left[\mu\left(\nabla \vec{v}+\nabla \vec{v}^{T}\right)\right]+\rho \vec{g}+\vec{F}$

Where $p$ is the static pressure, $\rho \vec{g}$ is the gravitational body force and $\vec{F}$ is the external body force defined by source terms.

Considering the significance of the surface tension, the continuum surface force (CSF) model [16] was introduced. Then the surface tension can be expressed by a volume force $F_{v}$ across the spreading interface, the force was added to the VOF model through the source terms of the momentum equation, it can be given as follows [16].

$$
F_{v}=\gamma_{i j} \frac{2 \rho k \nabla \alpha_{i}}{\left(\rho_{i}+\rho_{j}\right)}
$$

Where $\gamma_{i j}$ is the surface tension between two phases, $n=\nabla \alpha_{i}$ is the surface normal, $k=\nabla \cdot \hat{n}$ is the surface curvature, and $\hat{n}=n /|n|$ is the unit normal vector to the interface. Moreover, a wall adhesion term is introduced for specifying $\hat{n}$ at the liquid-solid-gas interface [16]: 


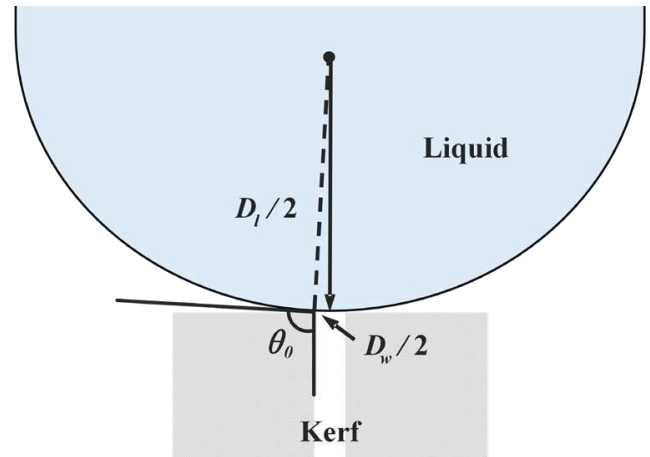

Fig. 4 Schematic of the initial contact angle when liquid just reaches the kerf.

$$
\hat{n}=\hat{n}_{w} \sin \theta_{d}+\hat{t}_{w} \cos \theta_{d}
$$

Where $\hat{n}_{w}$ and $\hat{t}_{w}$ are the unit vectors normal and tangential to the solid wall. $\theta_{d}$ is the dynamic contact angle between liquid and wall. Based on Hoffman's systematic study [17] of $\theta_{d}$ in glass capillary tubes, Jiang et al. [18] deduced an empirical correlation which fits the data equally well:

$$
\frac{\cos \theta_{d}-\cos \theta_{s}}{\cos \theta+1}=-\tanh \left(4.96 C a^{0.702}\right)
$$

Where $\theta_{s}$ is the static contact angle between liquid and wall. In this paper, the free liquid jet flows from the top to the kerf, hence, $\theta_{s}$ is assumed to be similar to the initial contact angle $\theta_{0}$ when liquid just reaches the kerf. As shown in Fig. 4, $\theta_{0}$ could be defined as: $\theta_{0} \approx 90^{\circ}+\arcsin \left(D_{\mathrm{w}} / D_{l}\right)$. Since $D_{w}$ is much smaller than the diameter of liquid jet $D_{l}=0.004, \theta_{s} \approx \theta_{0} \approx 90^{\circ}$. Then Eq. (10) is reduced as Eq. (11), and it was introduced to characterize the dynamic contact angle.

$$
\cos \theta_{d}=-\tanh \left(4.96 C a^{0.702}\right)
$$

The computational model was implemented into the Ansys Fluent software for calculation. The momentum equations were solved by the pressure-based solver. Meanwhile, the continuity equation was solved through implicit time discretization. For pressure velocity coupling, a PISO (Pressure Implicit Splitting of Operators) scheme [19] is used. For calculation accuracy, the global Courant number is set to 0.2 by adaptive time step. In addition, dynamic contact angle is introduced using UDF (User Defined Functions).

The following assumptions are made before the simulation setup: (1) both air and liquid were taken as incompressible fluids. (2) the motion of the wire was not considered, and the subface of the kerf is seen as a stationary plane. (3) this study focuses on the liquid flow regimes in the sawn kerf, heat transfer was not taken into account, and the entire process was assumed to be thermostatic at a temperature of $298.15 \mathrm{k}$.

\section{Results}

\subsection{The liquid entering-spreading progress}

Simulation research was first carried out with following parameters close to the general situation: $D_{i}=50.8 \mathrm{~mm}$, $A_{k}=1013.4 \mathrm{~mm}^{2}, D_{w}=0.3 \mathrm{~mm}, v_{i}=0.4 \mathrm{~m} / \mathrm{s}$ and the liquid properties of the solution $\mathrm{A}$.

The 3D liquid flow state outside the kerf is shown in Fig. 5(a). From Fig. 5a(i)-(ii), observe that the liquid flows from top to bottom first, then it comes into contact with the ingot. Gradually, a liquid layer is formed at the outer edge of the kerf (Fig. 5(a)- (iii-vi)). Moreover, the evolution of liquid contour in the center section of the kerf is shown in Fig. 5(b): as the liquid reaches the upper surface of the ingot (Fig. 5(b)-(i)), it enters the kerf (Fig. $5 \mathrm{~b}(\mathrm{ii})$ ) and spreads downward with an arc-shaped gas-liquid interface (Figs. 5(b)- (iii)). Then the spreading liquid reaches the kerf subface and covers the sawing area gradually (Fig. 5(b)- (iv)). Finally, it saturates the kerf and maintain an equilibrium state (Fig. 5(b)-(vi) and Fig. 5(b)-(vi)) when $T \geq 0.45 \mathrm{~s}$.

In order to evaluate the spreading behavior, the liquid flow time to reach the equilibrium state $t_{e}$ is taken as an evaluation index in the following section.

\section{2 Parametric simulation}

\subsubsection{Effect of kerf area}

Fig. 6 shows the simulation results of various $A_{k}$. The evolution of liquid contours is presented with the scatter plot of $t_{e}$. It is noted that with the increase of $A_{k}$, the liquid spreading interface gradually transforms from arc-shape to U-shape. But actually, there is no fundamental discrepancy in the liquid spreading regimes: the liquid, flowing into the kerf from top to bottom, spreads to both sides after arriving at the center of the sawing area, and finally reaches an equilibrium state. Moreover, the results of $A_{k 4}$ and $A_{k 5}$ shows that, the kerf cannot be filled after the liquid contour is stable (Fig. 6(a-3)-v and (a-4)-v), which is attribute to the insufficient liquid flow. Further, observe that $t_{e}$ increase significantly with $A_{k}$ (Fig. 6), which also indicates that greater liquid flow is required for large $A_{k}$. 
(a)

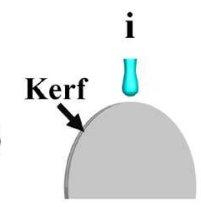

(b)

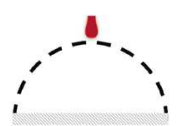

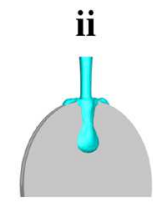

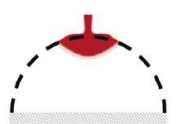

0.09
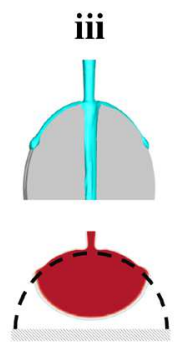

0.27
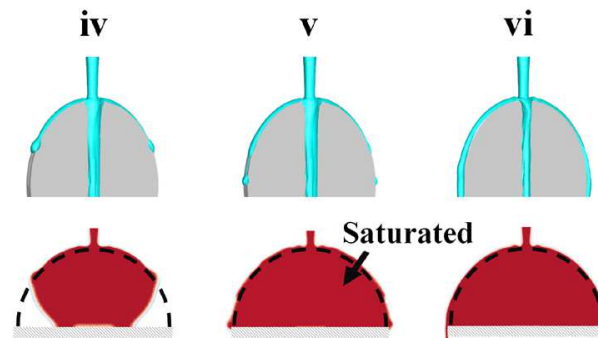

0.35

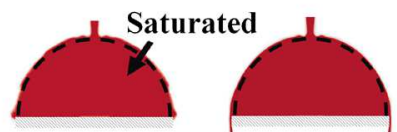

0.45
0.76

Figure 5 The liquid entering-spreading process in the kerf (a) 3D plot of the liquid dynamic flow state, (b) transient evolution of the liquid contours in the center section of the kerf. Simulation parameters: $D_{i}=50.8 \mathrm{~mm}, A_{k}=1013.4 \mathrm{~mm}^{2}, D_{w}=0.3 \mathrm{~mm}, v_{i}=0.67 \mathrm{~m} / \mathrm{s}$, liquid properties of solution A.

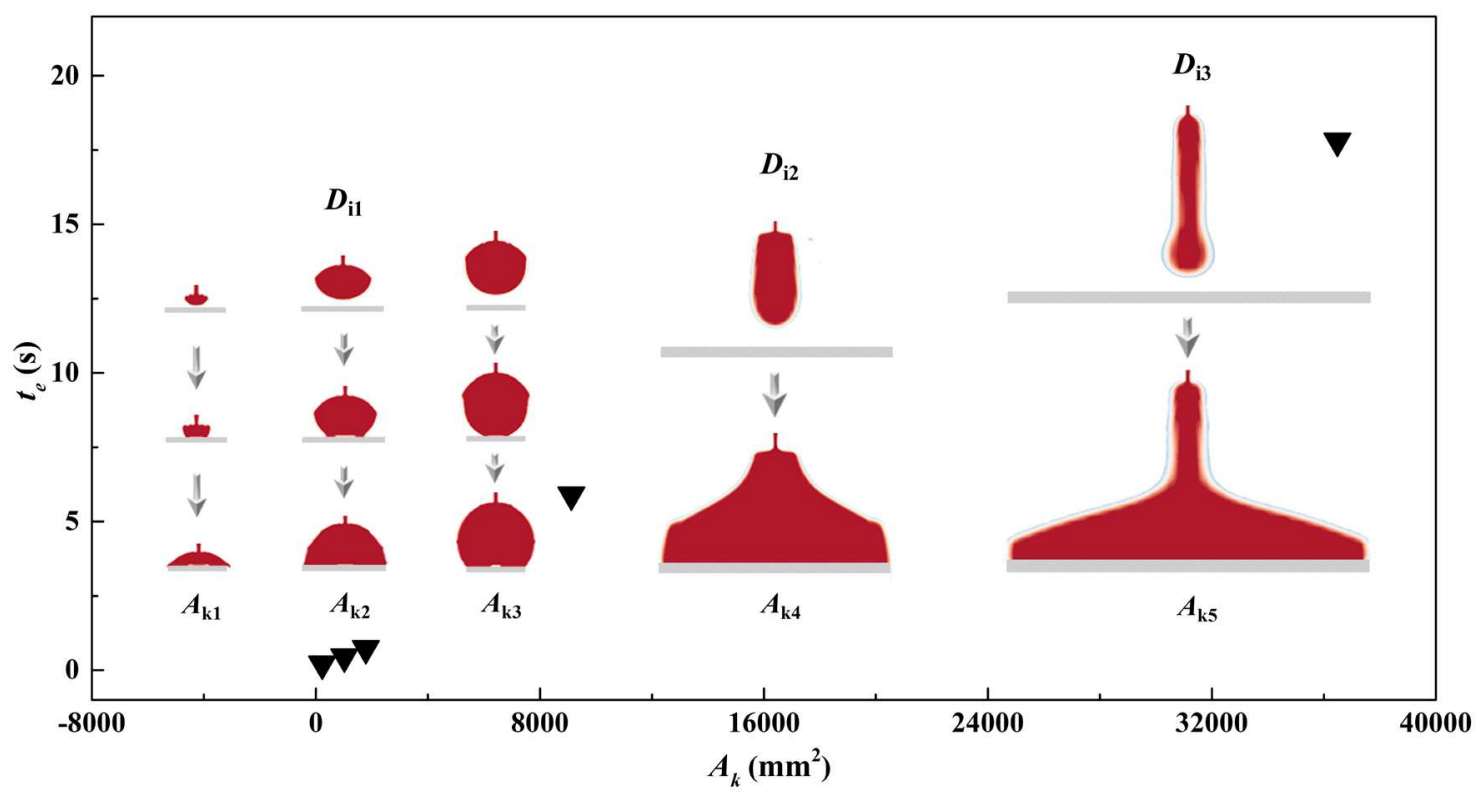

Figure 6 Plot of the liquid flow time to reach equilibrium with different kerf areas and the evolution of liquid spreading contour $\left(A_{k 1}=\right.$ $223.14 \mathrm{~mm}^{2}, T=[0.07,0.11,0.21] \mathrm{s} ; A_{k 2}=1013.4 \mathrm{~mm}^{2}, T=[0.27,0.35,0.45] \mathrm{s} ; A_{k 3}=1782.94 \mathrm{~mm}^{2}, T=[0.49,0.58,0.74] \mathrm{s}$; $A_{k 4}=9120.73 \mathrm{~mm}^{2}, T=[1.92,5.95] \mathrm{s} ; A_{k 5}=35342.91 \mathrm{~mm}^{2} ; T=[2.71,17.80] \mathrm{s}$; other simulation parameters: $D_{w}=0.3 \mathrm{~mm}, v_{i}=$ $0.67 \mathrm{~m} / \mathrm{s}$, liquid properties of the solution A).

\subsubsection{Effect of kerf width}

The simulation results of various $D_{w}$ is show in Fig. 7 . $t_{e}$ shows an increase trend as $D_{w}$ declines, and $t_{\mathrm{e}}$ increases even drastically when $D_{w}>D_{w 4}$. Obviously, the decrease of $D_{w}$ makes it more difficult for the liquid to spread in the kerf. Meanwhile, the spreading contours present clear discrepancy in cases of various $D_{w}$. In cases $D_{w 1}, D_{w 2}$ and $D_{w 3}$, the liquid spreads with arc-shaped gas-liquid interfaces with different curvatures. However, in cases $D_{w 4}$ and $D_{w 5}$, distinct air chambers are noted in the kerf.

The formation process of air chamber phenomenon is shown in Fig. 8. Observe that, from the detail results of case $D_{w 5}$, when liquid enters the kerf, it spreads toward the both side of the kerf (Figs. 8(a-b)-(i)). Then the incoming liquid slides along the outer edge of the kerf (Figs. 8 (a-b)-ii). Gradually, a small amount of liquid seep into the kerf (Fig. 8(b)-iii), and the kerf is wrapped by the liquid layer, then there forms an air chamber in the kerf. Further, as the liquid spreads slowly and squeezes downward, there is an opening in the wrapped liquid layer (Figs. 8(a-b)-iv), then the air is squeezed out. Thus, the liquid spreads to the kerf subface and expels the air gradually (Fig. 8(b)-v). Finally, it reaches an equilibrium state (Fig. 8(b)-vi). 


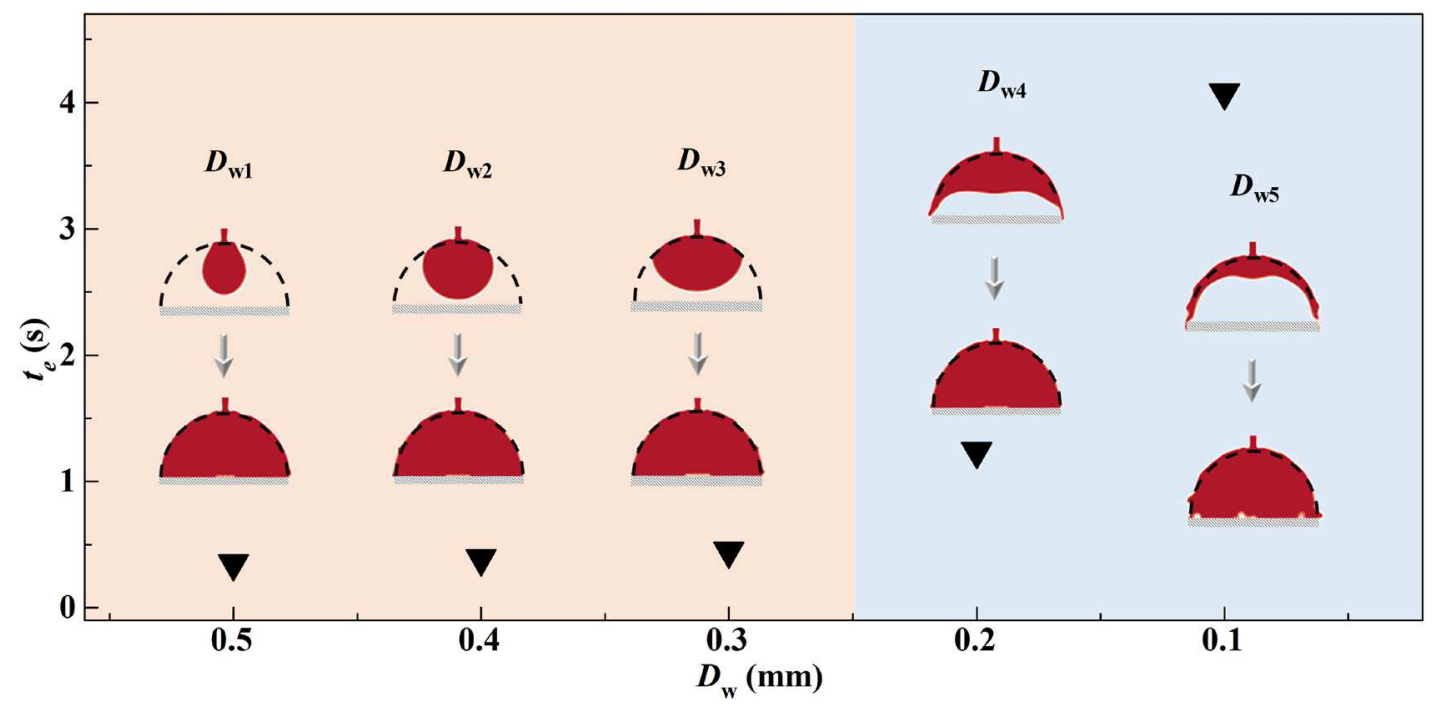

Figure 7 Plot of the liquid flow time to reach equilibrium with different kerf width and the evolution of liquid spreading contour $\left(D_{w 1}=0.5 \mathrm{~mm}, T=[0.14,0.35] \mathrm{s} ; D_{w 2}=0.4 \mathrm{~mm}, T=[0.20,0.39] \mathrm{s} ; D_{w 3}=0.3 \mathrm{~mm}, T=[0.30,0.45] \mathrm{s} ; \quad D_{w 4}=0.2 \mathrm{~mm}, T=[0.45\right.$, $1.04] \mathrm{s} ; \quad D_{w 5}=0.1 \mathrm{~mm} ; T=[0.35,4.08] \mathrm{s}$; other simulation parameters: $A_{k 2}=1013.4 \mathrm{~mm}^{2}, \quad v_{i}=0.67 \mathrm{~m} / \mathrm{s}$, liquid properties of solution A).

(a)
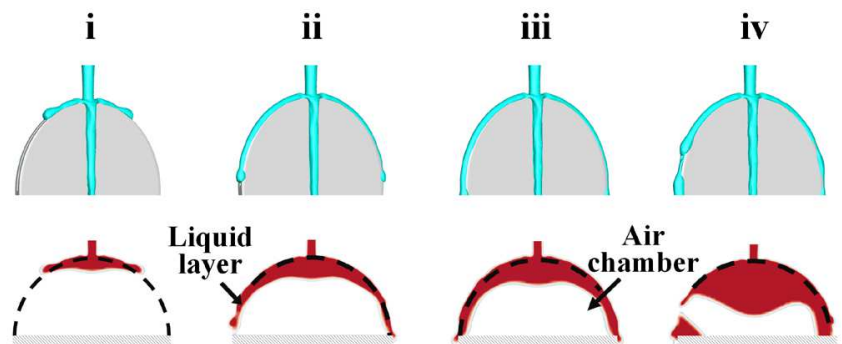

(b)

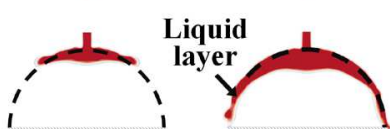

0.31

0.45

1.86
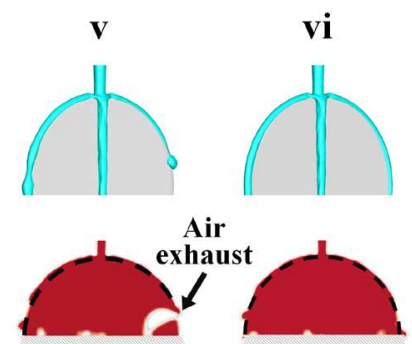

$T(\mathbf{s})$

0.14

Figure 8 Results of simulation with the kerf width of $0.1 \mathrm{~mm}$ : (a) 3D plot pf the liquid dynamic flow regime, (b) Transient evolution of the liquid contours in the center section of the kerf (Other simulation parameters: $D_{i}=50.8 \mathrm{~mm}, A_{k}=1013.4 \mathrm{~mm}^{2}, D_{w}=0.3 \mathrm{~mm}$, $v_{i}=0.4 \mathrm{~m} / \mathrm{s}$, liquid properties of solution A).

\subsubsection{The effect of liquid viscosity}

Simulation results of various liquid viscosity $\mu_{l}$ is presented in Fig. 9. Plotted with the liquid spreading contours, $t_{e}$ shows an accelerated increase trend with $\mu_{l}$. Moreover, a sharp increase in $t_{e}$ is seen between $\mu_{l 2}$ and $\mu_{l 3}$ (as indicated by the red arrow). This could be attribute to the formation of air chamber phenomenon when $\mu_{l} \geq \mu_{l 3}$ (see Fig. 9). By comparing the liquid spreading area in the same flow time $(0.3 \mathrm{~s})$, it is confirmed that the grow of $\mu_{l}$ will significantly reduce the liquid spreading rate in the kerf, and it will also contribute to the air chamber phenomenon.

\subsubsection{The effect of liquid surface tension}

The profile of $t_{e}$ with various liquid surface tension $\gamma_{l v}$ is shown in Fig. 10. There is an upward trend in $t_{e}$ as $\gamma_{l v}$ increases, and a growth spurt is seen between $\gamma_{l v 3}$ and $\gamma_{l v 4}$. Moreover, as $\gamma_{l v}$ increases, the spreading interface gradually evolves from an arc-shape $\left(\gamma_{l v 1}\right)$ to a flatter shape $\left(\gamma_{l v 2}\right.$ and $\left.\gamma_{l v 3}\right)$, further forming a liquid layer that wraps the kerf, and there occurs the air chamber in the kerf $\left(\gamma_{l v} \geq \gamma_{l v 4}\right)$. Therefore, it is speculated that the different spreading regimes could transform mutually when parameters are altered in a limit. In conclusion, it is found that the reduction of $\gamma_{l v}$ can facilitates the liquid 
spreading in the kerf, and it is helpful to avoid the formation of air chamber.

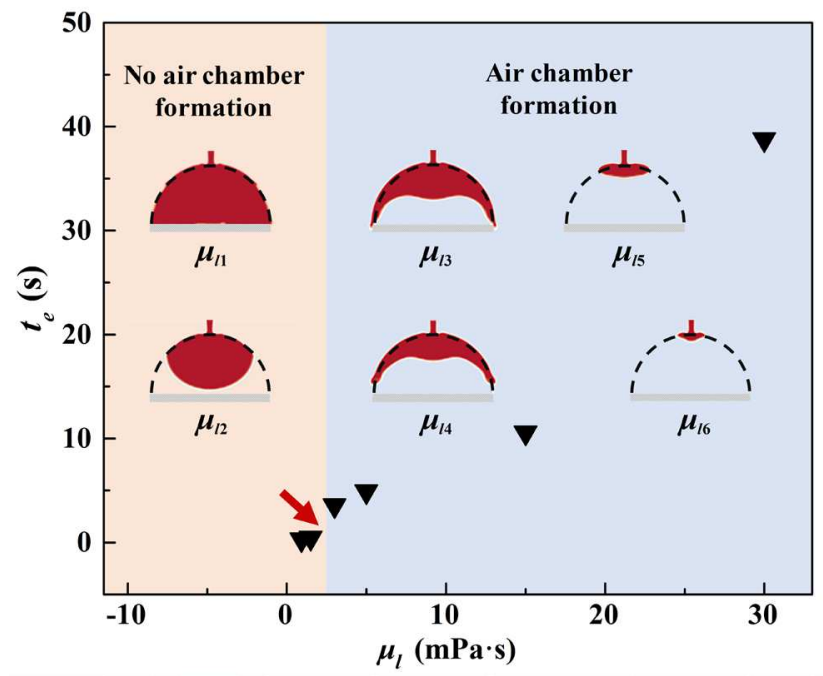

Figure 9 Plot of the liquid flow time to equilibrium with various liquid dynamic viscosity: $\mu_{l 1}=1 \mathrm{mPa} \cdot \mathrm{s}, \mu_{l 2}=1.5$ $\mathrm{mPa} \cdot \mathrm{s}, \mu_{l 3}=3 \mathrm{mPa} \cdot \mathrm{s}, \mu_{l 4}=5 \mathrm{mPa} \cdot \mathrm{s}, \mu_{l 5}=15 \mathrm{mPa} \cdot \mathrm{s}, \mu_{l 6}=$ $30 \mathrm{mPa} \cdot \mathrm{s}$. It also shows the liquid spreading contours at liquid flow time of $0.3 \mathrm{~s}$ (other simulation parameters: $D_{i}=50.8 \mathrm{~mm}$, $A_{k}=1013.4 \mathrm{~mm}^{2}, D_{w}=0.3 \mathrm{~mm}, v_{i}=0.67 \mathrm{~m} / \mathrm{s}, \quad \gamma_{l v}=50 \mathrm{mN} / \mathrm{m}$, $\left.\rho_{l}=999.12 \mathrm{~kg} / \mathrm{m}^{3}\right)$.

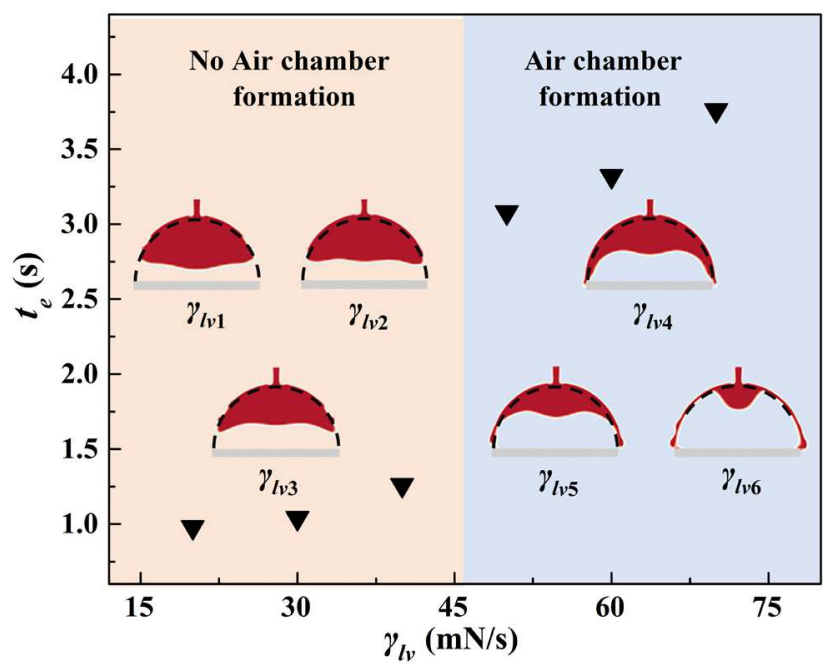

Figure 10 Plot of the time to the equilibrium state with various liquid surface tension: $\gamma_{l v 1}=20 \mathrm{mN} / \mathrm{m}, \quad \gamma_{l v 2}=30 \mathrm{mN} / \mathrm{m}, \quad \gamma_{l v 3}=$ $40 \mathrm{mN} / \mathrm{m}, \quad \gamma_{l v 4}=50 \mathrm{mN} / \mathrm{m},=60 \mathrm{mN} / \mathrm{m}, \quad \gamma_{l v 5}=70 \mathrm{mN} / \mathrm{m}$. It also shows the liquid spreading contours at liquid flow time of $0.3 \mathrm{~s}$ (other simulation parameters: $D_{i}=50.8 \mathrm{~mm}, A_{k}=1013.4 \mathrm{~mm}^{2}$, $\left.D_{w}=0.3 \mathrm{~mm}, \quad v_{i}=0.67 \mathrm{~m} / \mathrm{s}, \quad \mu_{l}=3 \mathrm{mPa} \cdot \mathrm{s}, \quad \rho_{l}=999.12 \mathrm{~kg} / \mathrm{m}^{3}\right)$.

\subsubsection{Effect of liquid inlet velocity}

Simulation results of various liquid velocity $v_{i}$ is presented in Fig. 11. Plotted with the liquid spreading contours, $t_{e}$ shows an accelerated increase trend as the decrease of $v_{i}$. Moreover, a special phenomenon is observed when $v_{i}=v_{i 3}$ : almost no liquid could flow into the kerf, then $t_{\mathrm{e}}$ can be considered infinite. Further, as $v_{i}$ decreases, the evolution of the spreading interface is observed again: the spreading interface gradually evolves from an arc-shape $\left(v_{i 1}\right)$ to a liquid layer wrapping the kerf $\left(v_{i 2}\right.$ and $\left.v_{i 3}\right)$, and eventually, the liquid can barely enter the $\operatorname{kerf}\left(v_{i 4}\right)$.

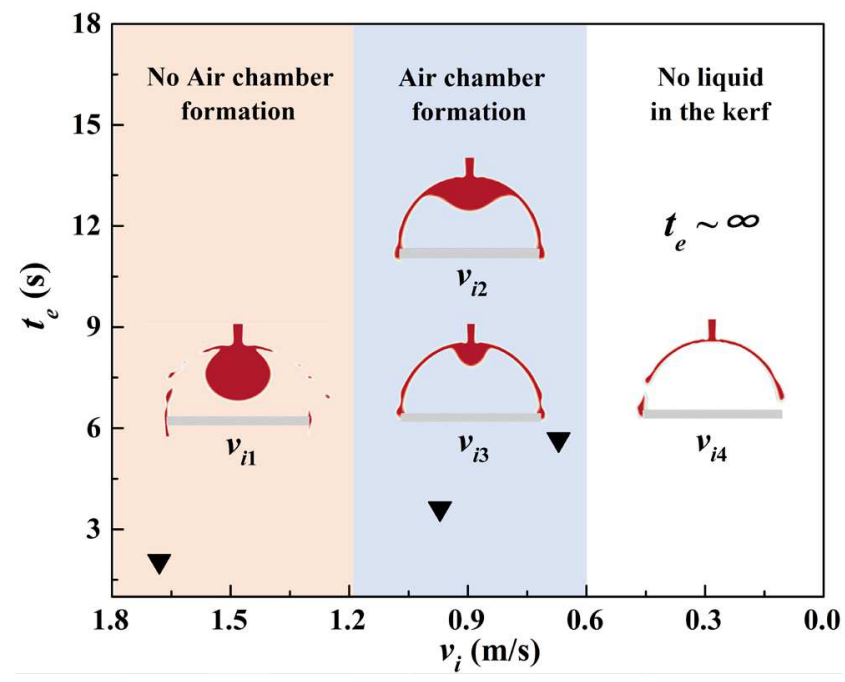

Figure 11 Plot of the time to the equilibrium state with various liquid velocity at the inlet area of the kerf: $v_{i 1}=1.68, v_{i 2}=0.97$ $\mathrm{m} / \mathrm{s}, v_{i 3}=0.67 \mathrm{~m} / \mathrm{s}, v_{i 4}=0.55 \mathrm{~m} / \mathrm{s}$. It also shows the liquid spreading contours at liquid flow time of $0.4 \mathrm{~s}$ (other simulation parameters: $D_{i}=50.8 \mathrm{~mm}, A_{k}=1013.4 \mathrm{~mm}^{2}, D_{w}=0.2 \mathrm{~mm}$, $\left.\mu_{l}=3 \mathrm{mPa} \cdot \mathrm{s}, \quad \gamma_{l v}=80 \mathrm{mN} / \mathrm{m}, \quad \rho_{l}=999.12 \mathrm{~kg} / \mathrm{m}^{3}\right)$.

\section{Discussion}

In the preceding sections 3 , numerical simulations were conducted to obtain the liquid spreading regime in the kerfs. By comparing the transient evolution of the liquid spreading contour, three typical liquid spreading regimes are found: (I) the liquid spreads up-down with an arc-shaped interface; (II) the spreading liquid wraps the outer contour of the kerf, forming an air chamber in the kerf; (III) the liquid could barely enter the kerf.

\subsection{Experimental verification}

Experimental verifications were conducted focusing on the three liquid spreading regimes. The experiments parameters are basically the same as that of simulations. 
As plot in Figs. 12 (a)-(b), by comparing the simulation and experimental results of the solution A, very similar evolution of liquid spreading contour with an arc-shaped spreading interface is observed with corresponding flow time. This confirms the authenticity of the liquid spreading regime I.

As shown in Figs. 13(a)-(b), similar liquid spreading evolution process can be notable observed, and the air

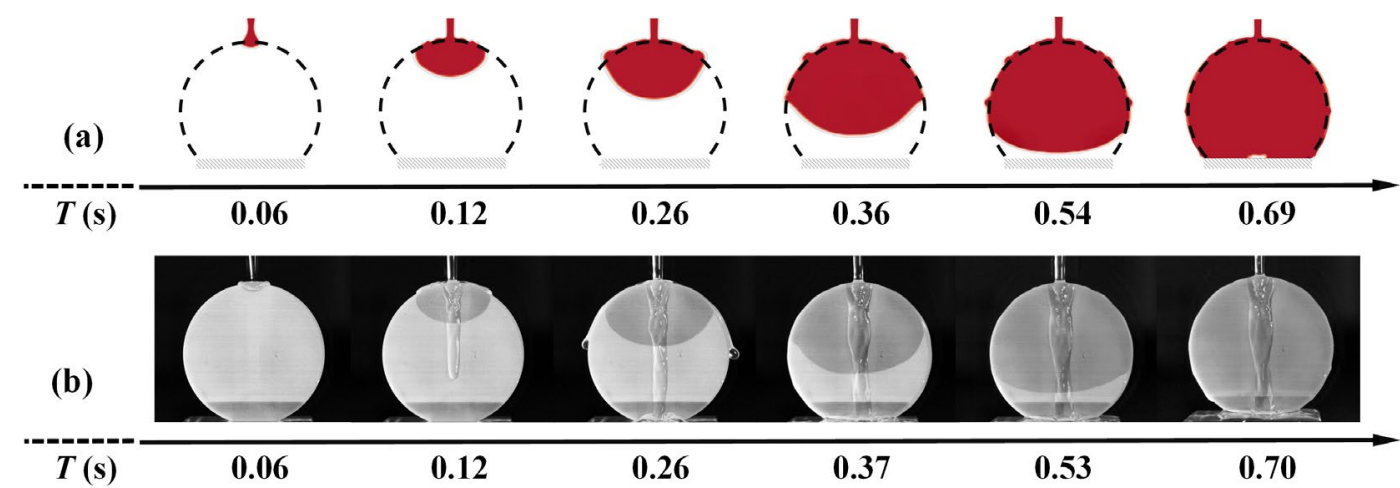

Figure 12 Transient evolution of the spreading contours of solution A in the kerf: (a) simulation results $\left(A_{k}=1782.94 \mathrm{~mm}^{2}, D_{i}=50.8\right.$ $\left.\mathrm{mm}, D_{w}=0.25 \mathrm{~mm}, \quad v_{i}=0.67 \mathrm{~m} / \mathrm{s}\right)$; (b) experimental results $\left(A_{k} \approx 1780.14 \mathrm{~mm}^{2}, D_{i}=50.8 \mathrm{~mm}, D_{w}=0.253 \mathrm{~mm}, \quad v_{i}=0.41 \mathrm{~m} / \mathrm{s}\right)$.

(a)

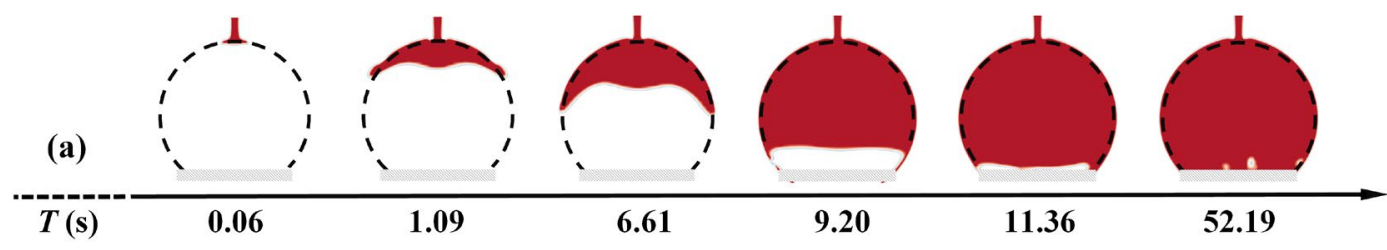

(b)
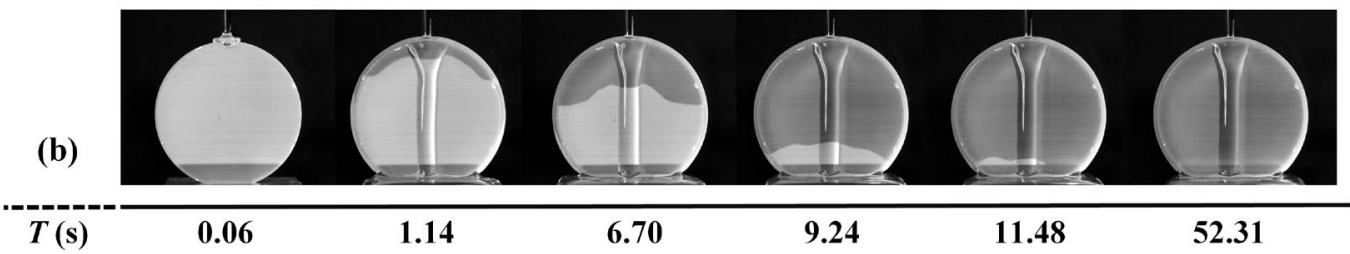

Figure 13 Transient evolution of the spreading contours of solution B in the kerf: (a) simulation results $\left(A_{k}=1782.94 \mathrm{~mm}^{2}, D_{i}=50.8\right.$ $\left.\mathrm{mm}, D_{w}=0.25 \mathrm{~m}, \quad v_{i}=0.67 \mathrm{~m} / \mathrm{s}\right) ;(\mathrm{b})$ experimental results $\left(A_{k} \approx 1780.14 \mathrm{~mm}^{2}, D_{i}=50.8 \mathrm{~mm}, D_{w}=0.253 \mathrm{~mm}, \quad v_{i}=0.403 \mathrm{~m} / \mathrm{s}\right)$.

(a)
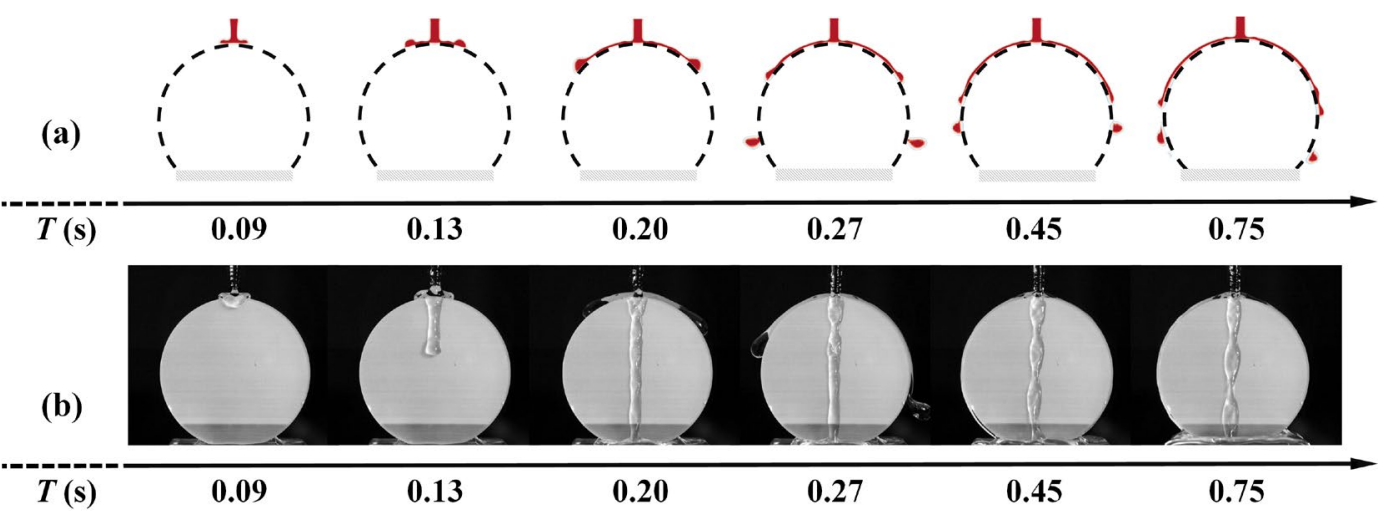

Figure 14 Transient evolution of the spreading contours of solution $\mathrm{C}$ in the kerf: (a) simulation results $\left(A_{k}=1782.94 \mathrm{~mm}{ }^{2}, D_{i}=50.8\right.$ $\left.\mathrm{mm}, D_{w}=0.25 \mathrm{~m}, v_{i}=0.55 \mathrm{~m} / \mathrm{s}\right) ;(\mathrm{b})$ experimental results $\left(A_{k} \approx 1780.14 \mathrm{~mm}^{2}, D_{i}=50.8 \mathrm{~mm}, \quad D_{w}=0.253 \mathrm{~mm}, \quad v_{i}=0.55 \mathrm{~m} / \mathrm{s}\right)$. 
(a)

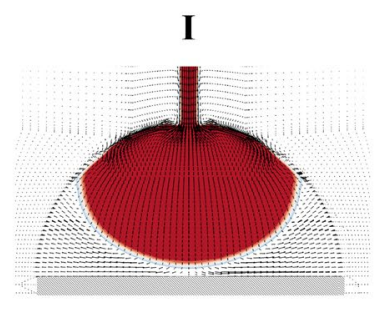

(b)

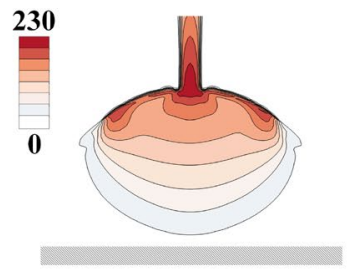

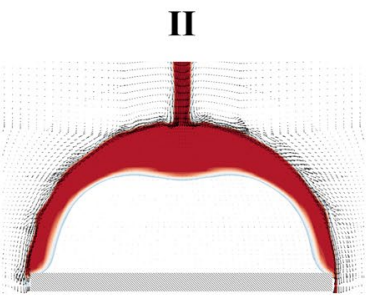
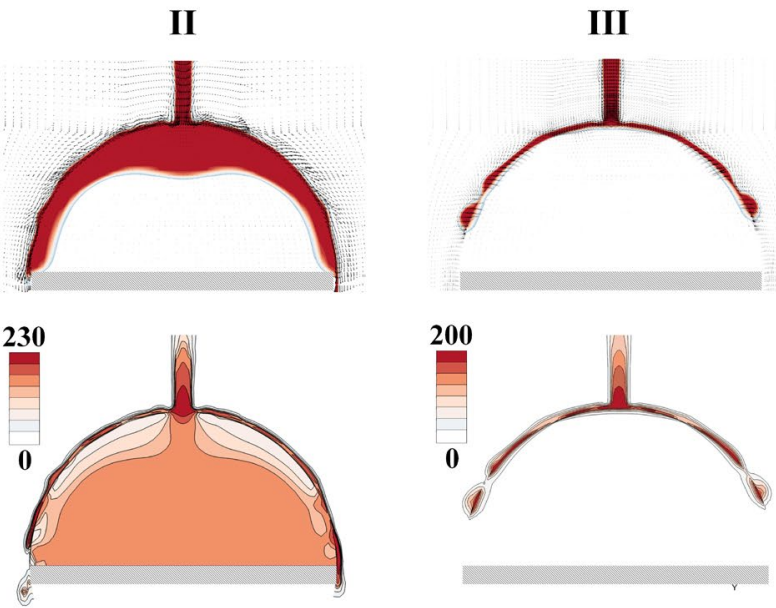

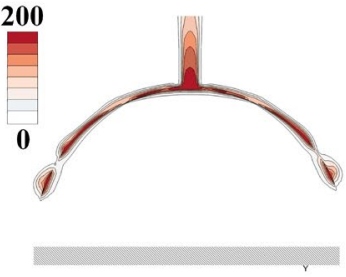

Figure 15 Three typical spreading regimes with corresponding (a) vector diagrams, (b) The total pressure nephograms. Simulation parameters: (I) $D_{i}=50.8 \mathrm{~mm}, A_{k}=1013.4 \mathrm{~mm}^{2}, D_{w}=0.3 \mathrm{~mm}, v_{i}=0.67 \mathrm{~m} / \mathrm{s}$, liquid properties of solution A, $T=0.3 \mathrm{~s}$; (II) $D_{i}=$ $50.8 \mathrm{~mm}, A_{k}=1013.4 \mathrm{~mm}^{2}, D_{w}=0.1 \mathrm{~mm}, \quad v_{i}=0.67 \mathrm{~m} / \mathrm{s}$, liquid properties of solution A, $T=0.45 \mathrm{~s}$; (III) $D_{i}=50.8 \mathrm{~mm}, A_{k}=1013.4$ $\mathrm{mm}^{2}, \quad D_{w}=0.2 \mathrm{~mm}, \quad v_{\mathrm{i}}=0.58 \mathrm{~m} / \mathrm{s}, \quad \mu_{l}=3 \mathrm{mPa} \cdot \mathrm{s}, \quad \gamma_{l v}=80 \mathrm{mN} / \mathrm{m}, \quad \rho_{l}=999.12 \mathrm{~kg} / \mathrm{m}^{3}, \quad T=0.3 \mathrm{~s}$.

chamber phenomenon is seen in both simulation and experimental results of the solution B. This confirms the existence of regime II.

As shown in Figs. 14(a)-(c), very similar evolution of liquid spreading contour is observed from both simulation and experimental results: the liquid can barely get into the kerf, and it just spreads around the outer edge of the kerf. It proves the authenticity of liquid spreading regime III.

\subsection{Causes and influences of spreading regimes}

To figure out the causes of different spreading regimes, typical simulation cases are selected, and the liquid contours, velocity vector and pressure in the kerf for a certain flow time are shown in Fig. 15.

For the spreading regime I, significant pressure gradient (about 230-0 Pa, see Fig. 15 (b)-I) is seen between the kerf top and the spreading interface, which could cause a large spreading velocity in the kerf [20]. It is performed by the significant velocity vector in the kerf, both in the liquid phase and air phase (Fig. 15 (a)-I). Thus, liquid could spread with an arc-shaped interface [21] in the kerf.

For the spreading regime II, the pressure gradient at the outer edge of the kerf is great (about 230-30 Pa, Fig. 15 (b)-II), where the velocity vector is significant. But the pressure gradient is much small between the kerf top and the spreading interface (about 230-170 Pa, see Fig. 15 (b)-II), where the velocity vector is almost invisible. Hence, the liquid is more likely to seep in from the outer edge and form a liquid layer wrapping the kerf, then an air chamber is generated.

For the spreading regime III, there are no liquid pressure gradient and velocity vectors in the kerf, as a result, liquid could barely enter the kerf.

Overall, in the spreading regime I, the liquid could spread in the kerf easier and quicker, then it expels air from the sawing area, infiltrates the processing area and achieve its important functions. However, in the spreading regime II, the air chamber badly hinders the spreading behavior of the liquid, even worse, it increases the probability of dry cutting in sawing. Moreover, in the spreading regime III, dry cutting is very likely to occur, and especially for MWSS, the abrasive could not be carried by liquid to the sawing area. Obviously, it is awfully detrimental to wire sawing.

\subsection{Non-dimensional and theoretical analysis}

It is found that, from Fig. 10 and 11, the different spreading regimes could transform mutually when parameters are altered in a limit. To figure out the mechanism, numerical simulations ware conducted extensively (all relevant simulation parameters and results are shown in appendix C).

Define that the Weber number and Capillary number representing each different simulation case as: $W e_{r}=\rho_{l} v_{i}^{2} l_{0} / \gamma_{l v}$ and $C a_{r}=\mu_{l} v_{i} / \gamma_{l v}$. Simulations were carried out within certain limits of $\mathrm{We}_{r} \quad(5 \sim 300)$ and $\mathrm{Ca}$ r $(0.3 \sim 8)$.

Comprehensive simulations results are potted in Fig. 16. The log-log plot shows that there are clear boundaries 


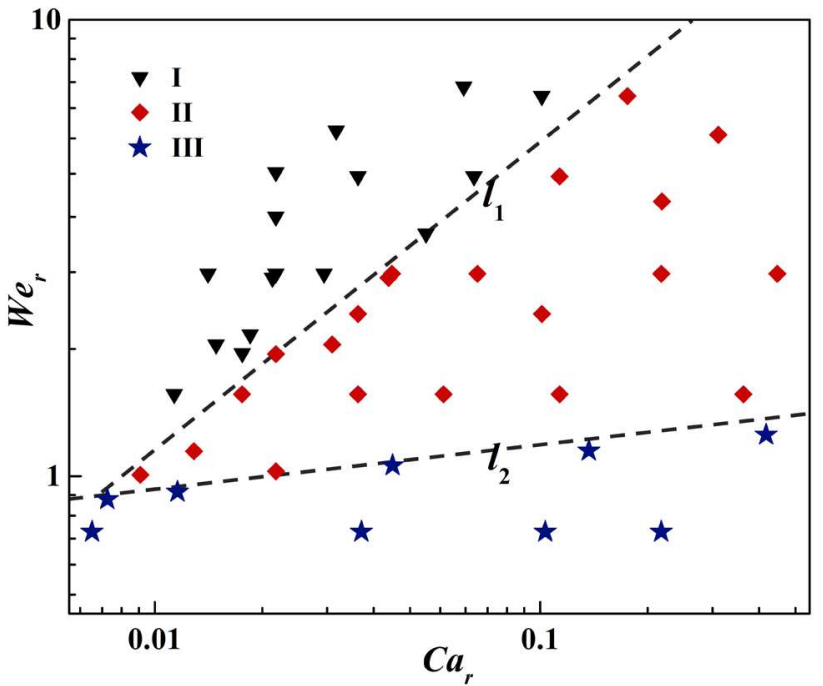

Figure 16 Non-dimensional map of the simulation results, which shows different liquid spreading regimes with the Weber number $W e_{r}$ on the horizonal axis and the Capillary number $\mathrm{Ca}$ on the vertical axis.

between the three spreading regimes. The two boundary lines are written by fitting the data in the boundary area (both with $R^{2} \approx 0.99$ ):

$$
\begin{aligned}
& l_{1}: W e_{r}=24.8 C a_{r}{ }^{0.65} \\
& l_{2}: W e_{r}=1.47 C a_{r}{ }^{0.1}
\end{aligned}
$$

Therefore, a predictive model is proposed to identify the liquid spreading regime, by given a set of Webb number and Capillary number:

$$
\left\{\begin{array}{l}
\text { Regime I : } W e_{r}>24.8 C a_{r}^{0.65} \\
\text { Regime II }: 1.47 C a_{r}^{0.1}<W e_{r}<24.8 C a_{r}^{0.65} \\
\text { Regime III }: W e_{r}<1.47 C a_{r}^{0.1}
\end{array}\right.
$$

This paper illuminated the influence of liquid spreading behavior in the kerf on wire sawing. It also provides a new thinking approach for solving problems which may encounter in wire sawing. Clearly, more work needs to be done to corroborate the application of the dynamic contact angle model in the 3D geometry. However, the high validity simulation method used in this paper may contribute to the research on the filling flow in small scale, such as fluid problems in precision machining, micro injection molding, etc.

\section{Conclusion}

(1) The present studies were designed to investigate the liquid spreading behavior in the wire sawn kerf. A CFD simulation model was established, and parametric simulations have been successful in identifying the effect of kerf area, kerf width, liquid viscosity, liquid surface tension and liquid velocity at the inlet area of the kerf.

(2) As far as the development trend of wire sawing is concerned, the increase in the wafer area will not change the liquid spreading regime in the kerf, but the reduction of the kerf width will significantly hinder the liquid spreading behavior. By adjusting the physical properties and supply conditions of the liquid, the spreading regime can be effectively converted to facilitate wire sawing.

(3) From both simulation and experimental results, three typical liquid spreading regimes are found: (I) the liquid spreads up-down with an arc-shaped interface; (II) the spreading liquid wraps the outer contour of the kerf, forming an air chamber in the kerf; (III) the liquid could barely get into the kerf. Moreover, the limiting conditions of the three spreading regimes are identified by non-dimensional analysis, then a prediction model is proposed for the liquid spreading regime, by given a set of Weber number and Capillary number: when $W e_{r}>24.8 \mathrm{Ca}_{r}^{0.65}$, liquid basically spreads in the regime $\mathrm{I}$; when $1.47 \mathrm{Ca}_{r}^{0.1}<\mathrm{We}_{r}<24.8 \mathrm{Ca}_{r}^{0.65}$, liquid basically spreads in the regime II; when $\mathrm{We}_{r}<1.47 \mathrm{Ca}_{r}^{0.1}$, liquid basically spreads in the regime III.

\section{Declaration}

\section{Funding}

Supported by the National Natural Science Foundation of China (NSFC) (Grant Nos. 51375179) from Changjiang Scholars and Innovative Research Team in University (IRT_17R41).

\section{Availability of data and materials}

The datasets supporting the conclusions of this article are included within the article.

\section{Authors' contributions}

The author' contributions are as follows: Hui Huang was in charge of the whole trial; Lin Lin wrote the manuscript; and finished sampling and laboratory analyses.

\section{Competing interests}


The authors declare no competing financial interests.

\section{Consent for publication}

Not applicable

\section{Ethics approval and consent to participate}

Not applicable

\section{References}

[1] H J Möller. Basic Mechanisms and Models of Multi-Wire Sawing. Advanced Engineering Materials, 2004, 6(7): 501-513. DOI: 10.1002/adem.200400578.

[2] $\mathrm{H} \mathrm{Wu}$. Wire sawing technology: A state-of-the-art review. Precision Engineering, 2016, 43: 1-9.

[3] M Shinya, T Yazawa, T Otubo. Study on the slicing of sapphire using a wire tool. Advanced Materials Research, 2015, 1136: 333-337.

[4] M Bhagavat, V Prasad, I Kao. Elasto-hydrodynamic interaction in the free abrasive wafer slicing using a wiresaw: modeling and finite element analysis. Journal of Tribology, 2000, 122(2): 394-404.

[5] F Yang, I Kao. Free Abrasive Machining in Slicing Brittle Materials with Wire saw. Journal of Electronic Packaging, 2001, 123(3): 254-259.

[6] S Bhagavat, I Kao. A finite element analysis of temperature variation in silicon wafers during wiresaw slicing. International Journal of Machine Tools and Manufacture, 2008, 48(1): 95-106.

[7] L Johnsen, J E Olsen, T Bergstrom, K Gastinger. Heat Transfer During Multiwire Sawing of Silicon Wafers. Journal of Thermal Science and Engineering Applications, 2012, 4(3): 31006-31013.

[8] K I Ishikawa, H Suwabe, S Itoh, M Uneda. A Basic Study of the Behavior of Slurry Action at Multi-Wire Saw. Key Engineering Materials, 2003, 238-239: 89-92.

[9] B Nassauer, A Hess, M Kuna. Numerical and experimental investigations of micromechanical processes during wire sawing. International Journal of Solids \& Structures, 2014, 51(14): 2656-2665.

[10] J H Ferziger, M Peric, A Leonard. Computational Methods for Fluid Dynamics. Physics Today, 1997, 50(3): 80-84.

[11] C J Schär. A generalization of Bernoulli's theorem. Atmos. Sci., 50, 1993: 1437-1443.

[12] Smith, G D. An Introduction to Fluid Dynamics[J]. Physics Bulletin, 1959, 10(1):13-13.

[13] R. W Fox, A T Mcdonald, P J Pritchard. Fox and McDonald's introduction to fluid mechanics. New Jersey: Wiley, 2011,

[14] V Srinivasan, A J Salazar, K Saito. Modeling the disintegration of modulated liquid jets using volume-of-fluid (VOF) methodology. Applied Mathematical Modelling, 2011, 35(8): 3710-3730.

[15] C W Hirt, B D Nichols. Volume of fluid (VOF) method for the dynamics of free boundaries. Journal of Computational Physics, 1989, 39(1): 201-225.

[16] J U Brackbill, D B Kothe, C A Zemach. Continuum method for modeling surface tension. Journal of Computational Physics, 1992, 100(2): 335-354.

[17] R L Hoffman. A study of the advancing interface. I. Interface shape in liquid-gas systems. J. Colloid Interface Sci., 50, 1975, pp. 228-241.

[18] Šikalo, Š., Wilhelm, H.-D., Roisman, I., Jakirlić, S. and Tropea, C. Dynamic contact angle of spreading droplets: experiments and simulations. Phys. Fluids, 2005, 17, 062103.

[19] Barton, I. E., 2015, Comparison of SIMPLE- and PISO-type algorithms for transient flows, International Journal for Numerical Methods in Fluids, 26(4), pp. 459-483.

[20] Travis, K. P., and Gubbins, K. E., 2000, "Poiseuille flow of Lennard-Jones fluids in narrow slit pores," The Journal of Chemical Physics, 112(4), pp. 1984-1994.

[21] H E White, R F Paton. Modern college physics. New York, Van Nostrand Reinhold, 1962.

\section{Biographical notes}

Lin Lin, born in 1993, is currently a master of engineering. He received his master degree from Huaqiao University, China, in 2020.

Tel: +86-17359899069; E-mail: linlin.rylynn@gmail.com

Hui Huang, born in 1974, is currently a professor at Huaqiao University, China. He received his $\mathrm{Ph}$. D degree from Nanjing University of Aeronautics and Astronautics, China, in 2002. His research interests include the machining of brittle material and the manufacturing of superabrasive tools.

Tel: +86-592-6162615; E-mail: huanghuihh@hotmail.com

\section{Appendix}

Appendix A Parameters of the geometric model

\begin{tabular}{ccc}
\hline$D_{i}(\mathrm{~mm})$ & $A_{k}(\mathrm{~mm})$ & $D_{w}(\mathrm{~mm})$ \\
\hline & 223.14 & 0.3 \\
50.8 & 1013.4 & $0.1,0.2,0.25,0.3,0.4,0.5$ \\
& 1782.94 & 0.3 \\
152.4 & 9120.73 & 0.3 \\
304.8 & 35342.91 & 0.3 \\
\hline
\end{tabular}

Appendix B Wire sawing conditions and parameters

\begin{tabular}{cc}
\hline \multicolumn{2}{c}{ Sawing conditions and parameters } \\
\hline Machine model & JXQ-1201 \\
Sawing method & Single wire sawing \\
Wire speed & $6 \mathrm{~m} / \mathrm{s}$ \\
Table speed & $0.15 \mathrm{~mm} / \mathrm{min}$ \\
Wire tension & $25 \mathrm{~N}$ \\
Sawing interval & $50 \mathrm{~m}-1050 \mathrm{~m}$ \\
Ingot diameter & 2-inch ingot with a length of $50 \mathrm{~mm}$ \\
Diamond wire saw & $\Phi 0.18 \mathrm{~mm}, 30-40 \mu \mathrm{m}$ dia. \\
Sawn depth & $42.381 \mathrm{~mm}$ \\
Sawn width & $0.223 \mathrm{~mm}$ \\
\hline
\end{tabular}

Appendix C Simulation parameters and results in the non-dimensional analysis ( $R$ refers to the spreading regimes in the kerf)

\begin{tabular}{cccccccc}
\hline $\begin{array}{c}D_{w} \\
(\mathrm{~m})\end{array}$ & $\begin{array}{c}\mu_{l} \\
(\mathrm{~N} / \mathrm{m} \cdot \mathrm{s})\end{array}$ & $\begin{array}{c}\gamma_{l_{v}} \\
(\mathrm{~N} / \mathrm{m})\end{array}$ & $\begin{array}{c}v_{i} \\
(\mathrm{~m} / \mathrm{s})\end{array}$ & $\begin{array}{c}\rho_{l} \\
\left(\mathrm{~kg} / \mathrm{m}^{3}\right)\end{array}$ & $W e_{r}$ & $C a_{r}$ & $R$ \\
\hline 0.0001 & 0.0015 & 0.049 & 0.67 & 999.12 & 0.925 & 0.021 & $\mathrm{II}$ \\
0.0002 & 0.0015 & 0.049 & 0.67 & 999.12 & 1.851 & 0.021 & $\mathrm{II}$ \\
0.0003 & 0.0015 & 0.049 & 0.67 & 999.12 & 2.777 & 0.021 & $\mathrm{I}$ \\
0.0004 & 0.0015 & 0.049 & 0.67 & 999.12 & 3.702 & 0.021 & $\mathrm{I}$ \\
0.0005 & 0.0015 & 0.049 & 0.67 & 999.12 & 4.628 & 0.021 & $\mathrm{I}$ \\
0.0003 & 0.0300 & 0.049 & 0.67 & 999.12 & 2.777 & 0.413 & $\mathrm{II}$ \\
\hline
\end{tabular}




\begin{tabular}{|c|c|c|c|c|c|c|c|}
\hline 0.0003 & 0.0150 & 0.049 & 0.67 & 999.12 & 2.777 & 0.206 & II \\
\hline 0.0003 & 0.0050 & 0.049 & 0.67 & 999.12 & 2.777 & 0.069 & II \\
\hline 0.0003 & 0.0030 & 0.049 & 0.67 & 999.12 & 2.777 & 0.041 & II \\
\hline 0.0003 & 0.0020 & 0.049 & 0.67 & 999.12 & 2.777 & 0.028 & I \\
\hline 0.0003 & 0.0015 & 0.049 & 0.67 & 999.12 & 2.777 & 0.021 & I \\
\hline 0.0003 & 0.0010 & 0.049 & 0.67 & 999.12 & 2.777 & 0.014 & I \\
\hline 0.0003 & 0.0015 & 0.030 & 0.67 & 999.12 & 4.536 & 0.034 & I \\
\hline 0.0003 & 0.0015 & 0.050 & 0.67 & 999.12 & 2.722 & 0.020 & I \\
\hline 0.0003 & 0.0015 & 0.070 & 0.67 & 999.12 & 1.944 & 0.014 & I \\
\hline 0.0003 & 0.0015 & 0.080 & 0.67 & 999.12 & 1.701 & 0.008 & I \\
\hline 0.0003 & 0.0015 & 0.080 & 0.55 & 999.12 & 1.139 & 0.007 & I \\
\hline 0.0003 & 0.0015 & 0.049 & 0.55 & 999.12 & 1.860 & 0.017 & I \\
\hline 0.0003 & 0.0015 & 0.049 & 0.58 & 999.12 & 2.043 & 0.018 & I \\
\hline 0.0003 & 0.0015 & 0.049 & 0.67 & 999.12 & 2.777 & 0.021 & I \\
\hline 0.0003 & 0.0015 & 0.049 & 0.96 & 999.12 & 5.713 & 0.030 & I \\
\hline 0.0003 & 0.0015 & 0.049 & 1.68 & 999.12 & 17.45 & 0.052 & I \\
\hline 0.0002 & 0.0100 & 0.060 & 0.67 & 999.12 & 1.512 & 0.112 & II \\
\hline 0.0002 & 0.0050 & 0.060 & 0.67 & 999.12 & 1.512 & 0.056 & II \\
\hline 0.0002 & 0.0030 & 0.060 & 0.67 & 999.12 & 1.512 & 0.034 & II \\
\hline 0.0002 & 0.0015 & 0.060 & 0.67 & 999.12 & 1.512 & 0.017 & II \\
\hline 0.0002 & 0.0010 & 0.060 & 0.67 & 999.12 & 1.512 & 0.011 & I \\
\hline 0.0003 & 0.0150 & 0.070 & 0.96 & 999.12 & 3.999 & 0.207 & II \\
\hline 0.0003 & 0.0150 & 0.060 & 0.96 & 999.12 & 5.599 & 0.290 & II \\
\hline 0.0003 & 0.0050 & 0.020 & 0.67 & 999.12 & 6.804 & 0.168 & II \\
\hline 0.0003 & 0.0050 & 0.030 & 0.67 & 999.12 & 4.536 & 0.112 & II \\
\hline 0.0003 & 0.0015 & 0.020 & 0.67 & 999.12 & 6.804 & 0.101 & I \\
\hline 0.0003 & 0.0015 & 0.030 & 0.67 & 999.12 & 4.536 & 0.067 & I \\
\hline 0.0003 & 0.0015 & 0.040 & 0.67 & 999.12 & 3.402 & 0.051 & I \\
\hline 0.0003 & 0.0015 & 0.050 & 0.67 & 999.12 & 2.722 & 0.040 & II \\
\hline 0.0003 & 0.0015 & 0.060 & 0.67 & 999.12 & 2.268 & 0.034 & II \\
\hline 0.0003 & 0.0015 & 0.070 & 0.67 & 999.12 & 1.944 & 0.029 & II \\
\hline 0.0002 & 0.0010 & 0.072 & 0.55 & 999.12 & 0.898 & 0.007 & III \\
\hline 0.0002 & 0.0015 & 0.072 & 0.55 & 999.12 & 0.923 & 0.011 & III \\
\hline 0.0002 & 0.0050 & 0.070 & 0.58 & 999.12 & 1.056 & 0.041 & III \\
\hline 0.0002 & 0.0150 & 0.065 & 0.58 & 999.12 & 1.137 & 0.133 & III \\
\hline 0.0002 & 0.0400 & 0.060 & 0.58 & 999.12 & 1.2328 & 0.386 & III \\
\hline 0.0002 & 0.0010 & 0.08 & 0.55 & 999.12 & 0.755 & 0.006 & III \\
\hline 0.0002 & 0.0050 & 0.08 & 0.55 & 999.12 & 0.755 & 0.034 & III \\
\hline 0.0002 & 0.0150 & 0.08 & 0.55 & 999.12 & 0.755 & 0.103 & III \\
\hline 0.0002 & 0.0300 & 0.08 & 0.55 & 999.12 & 0.755 & 0.206 & III \\
\hline
\end{tabular}



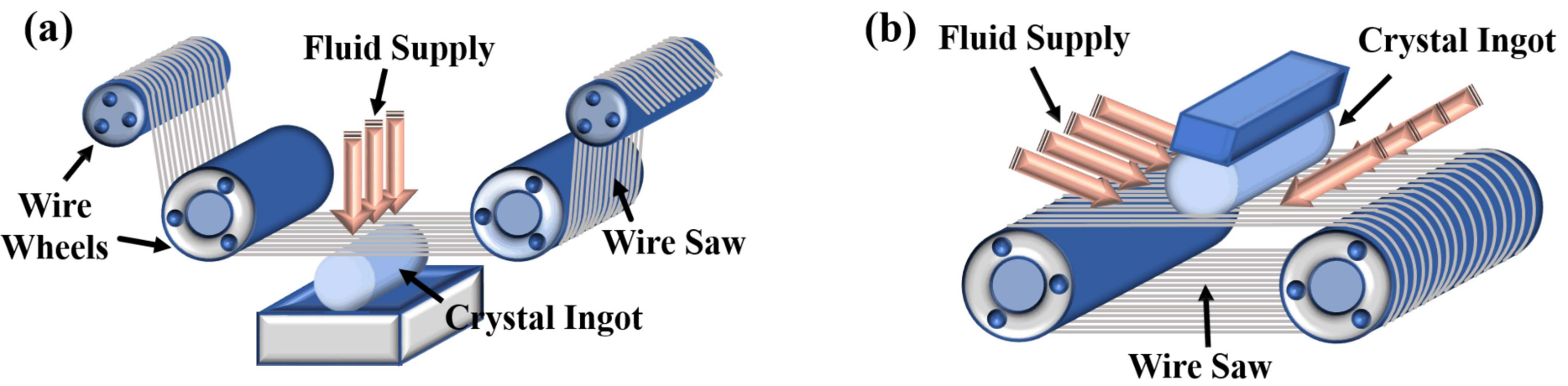

Figure 1

Two main liquid-supply modes in abrasive wire sawing: (a) spray into the kerf, (b) spray to the moving wire web.

(a)

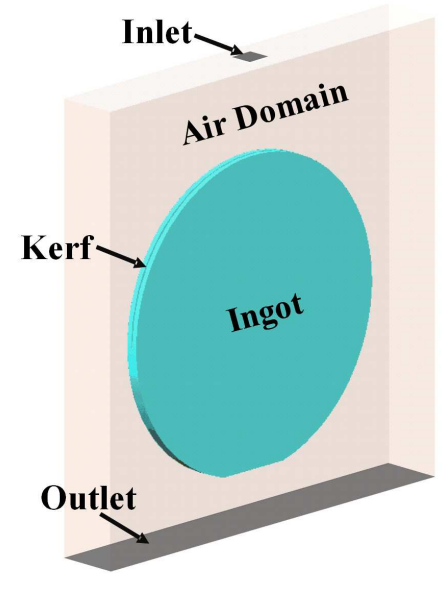

(b)

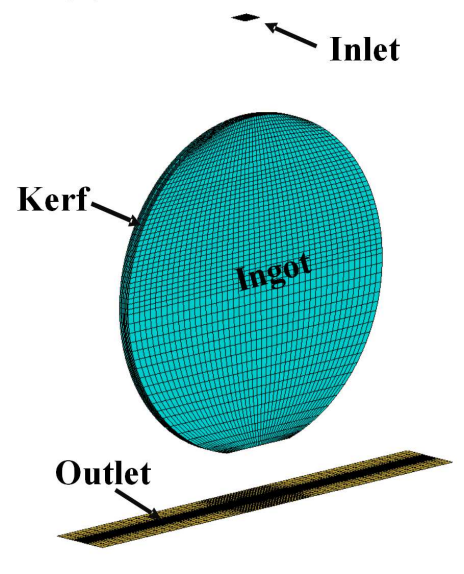

(c)
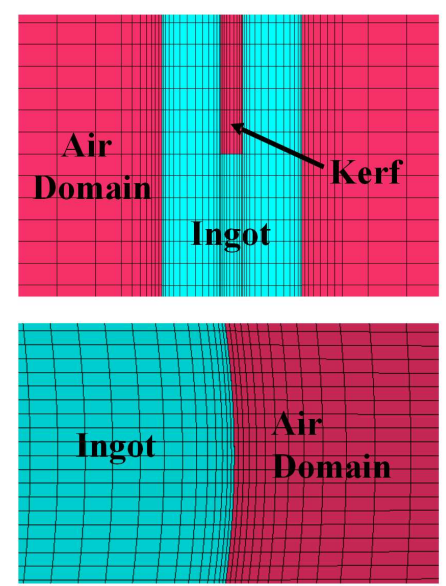

(d)
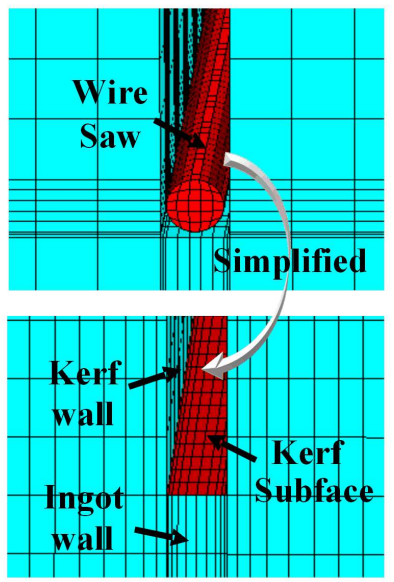

Figure 2

Geometric model and grids: (a) the calculation domain and geometric model, (b) the grid of the ingot with a kerf, (c) the local mesh densification at the wall of ingot and the kerf, (d) the simplification of the sawing area. 


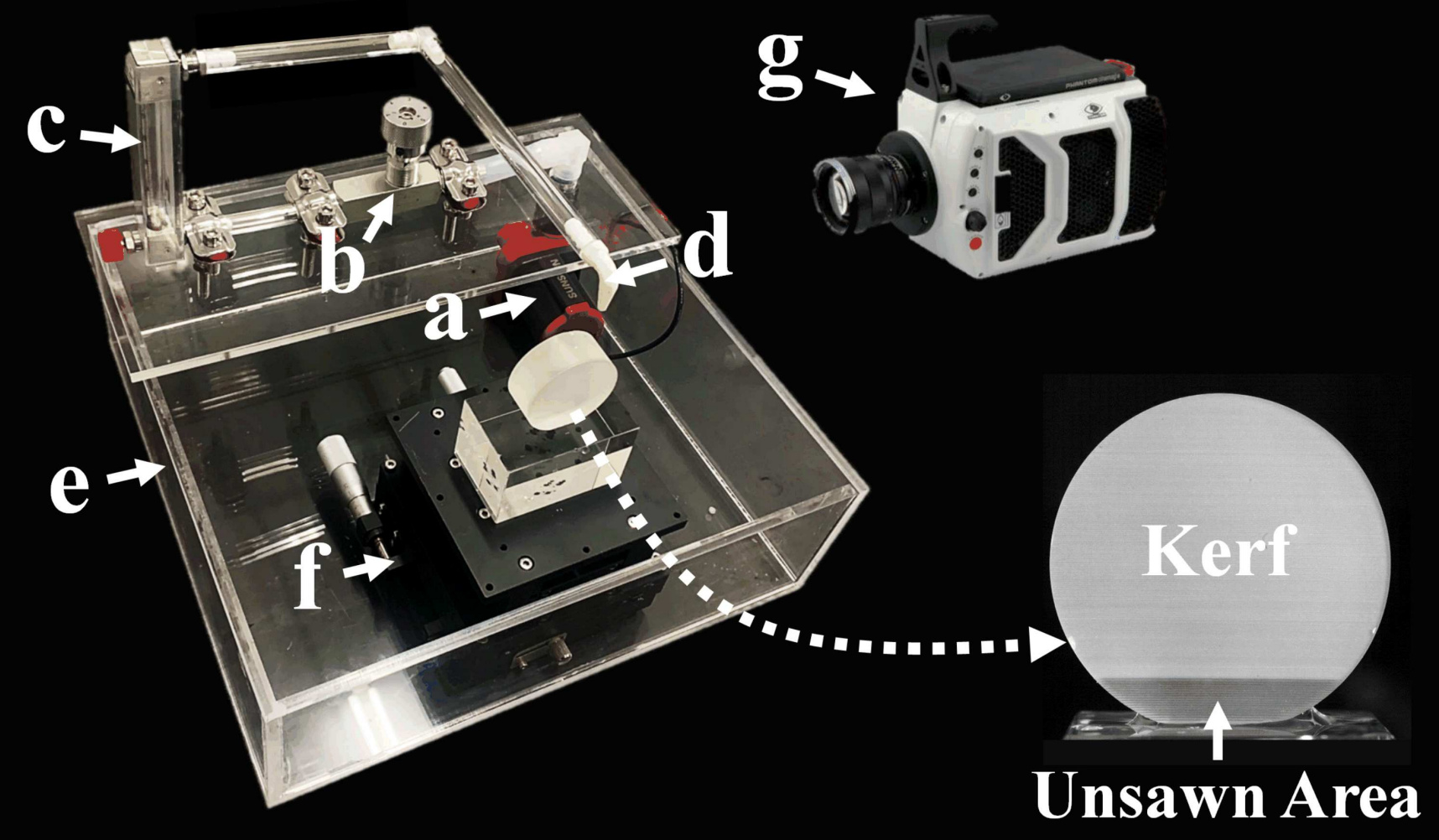

Figure 3

Experimental setup. 


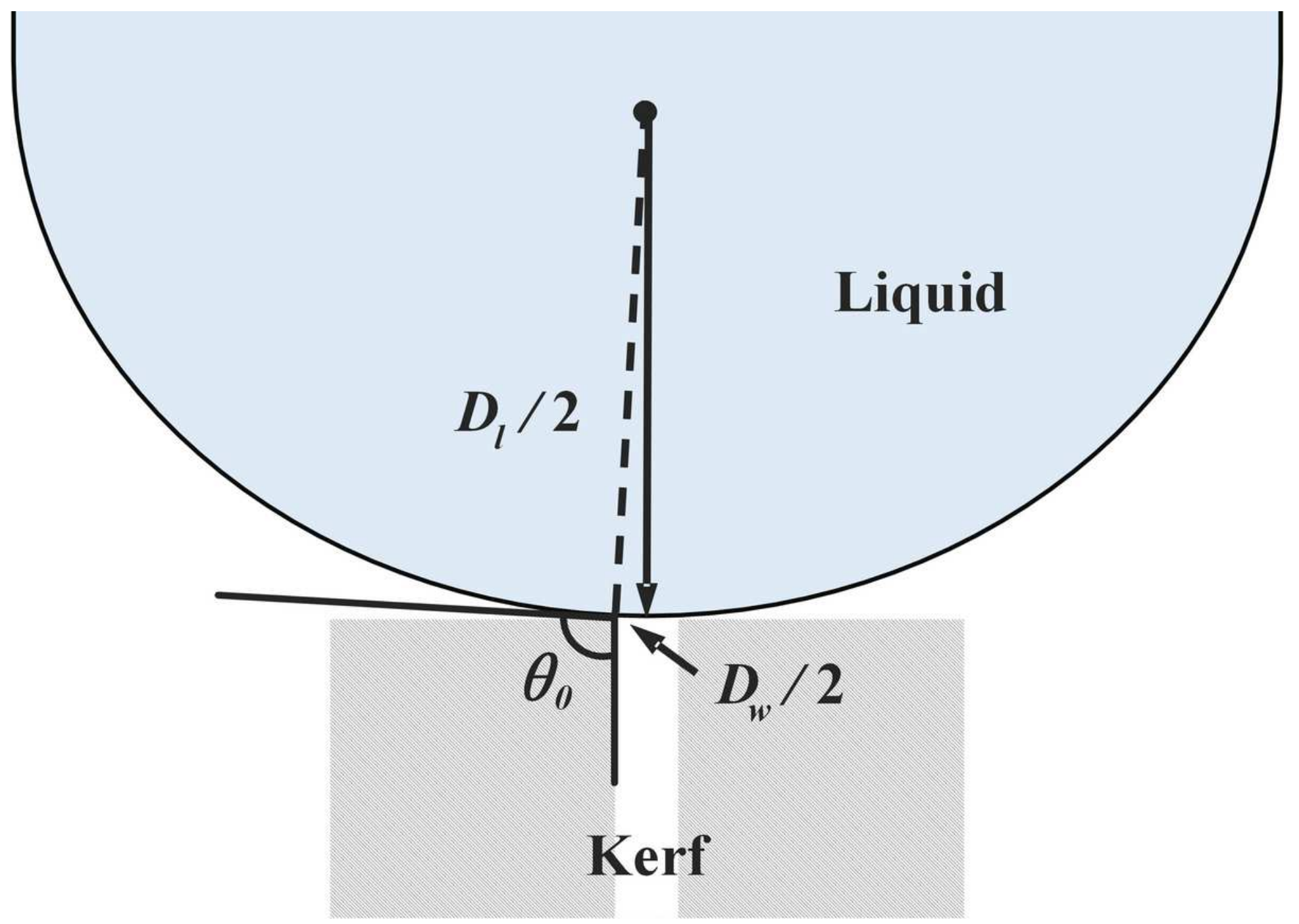

Figure 4

Schematic of the initial contact angle when liquid just reaches the kerf.

(a)
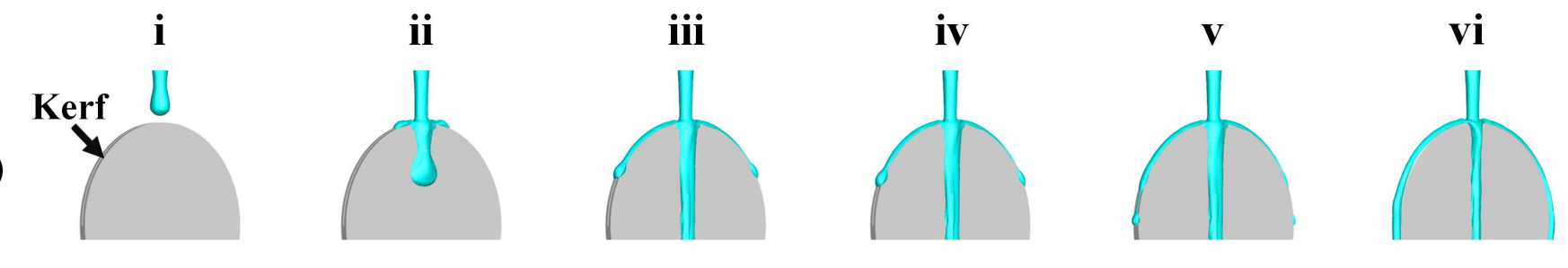

(b)
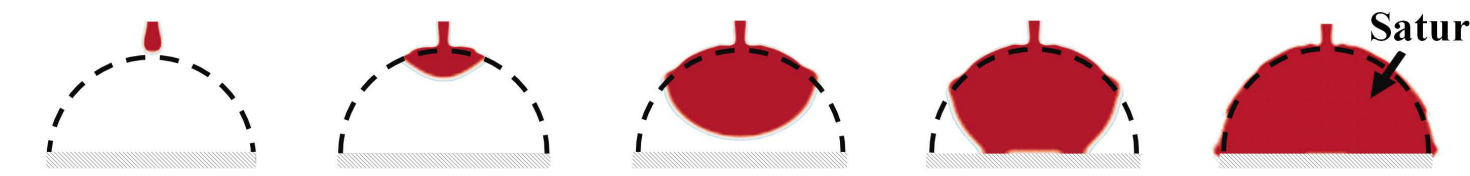

$$
T(\mathbf{s}) \quad 0.05
$$

0.09

0.27

0.35

0.45

0.76

Figure 5 


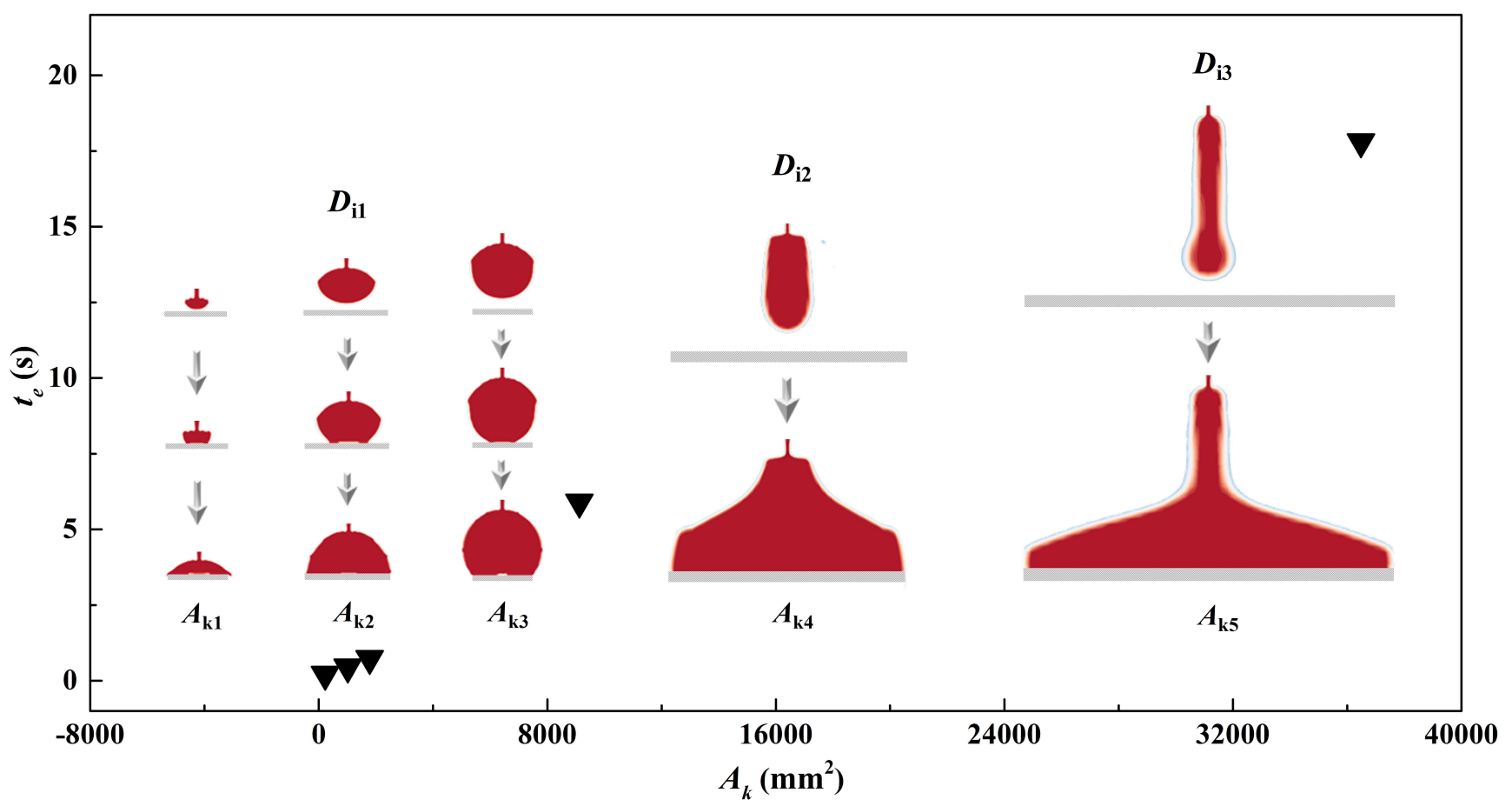

Figure 6

Plot of the liquid flow time to reach equilibrium with different kerf areas and the evolution of liquid spreading contour Please see manuscript .pdf for full caption

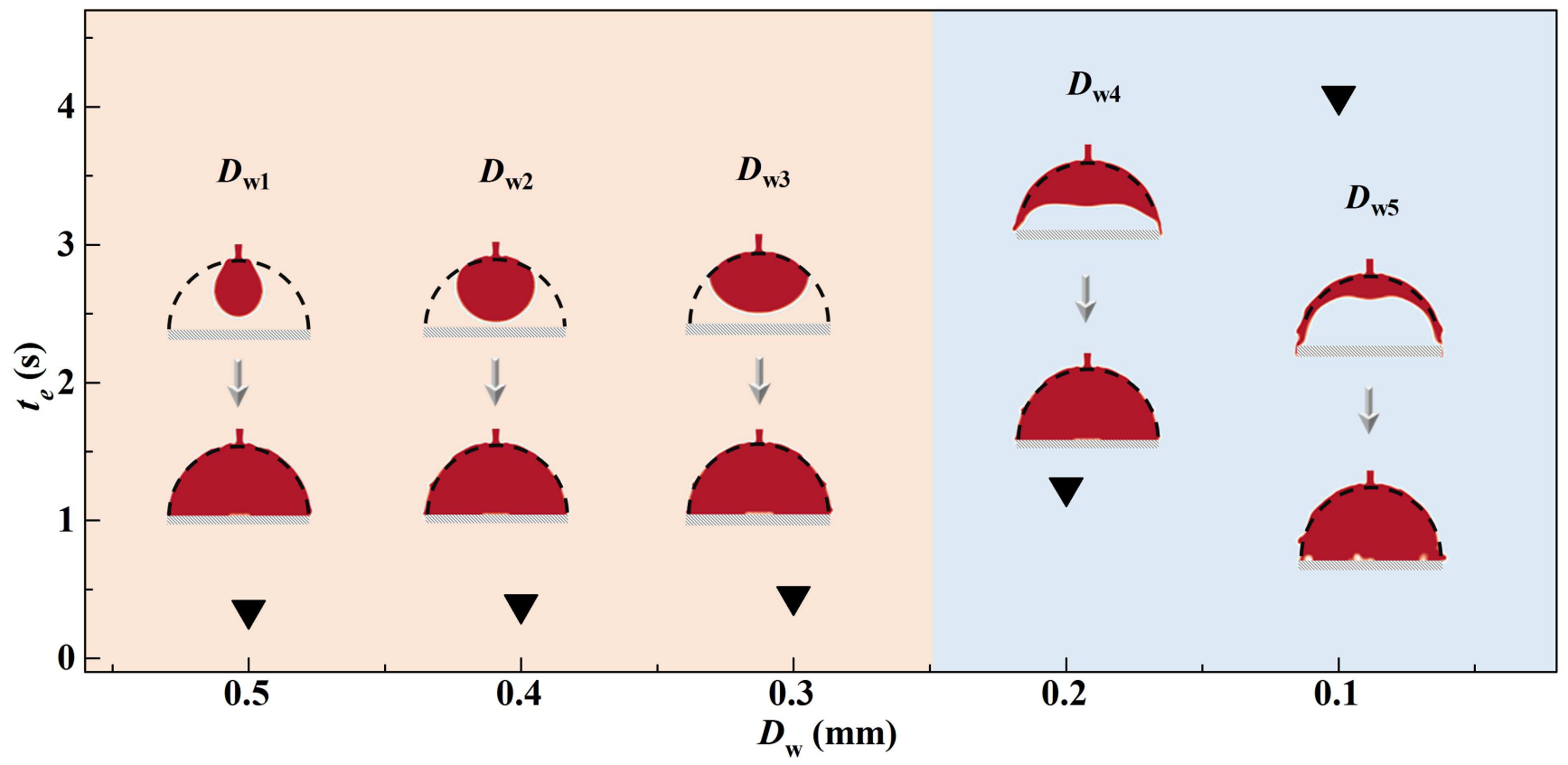

Figure 7 
Plot of the liquid flow time to reach equilibrium with different kerf width and the evolution of liquid spreading contour Please see manuscript .pdf for full caption

(a)
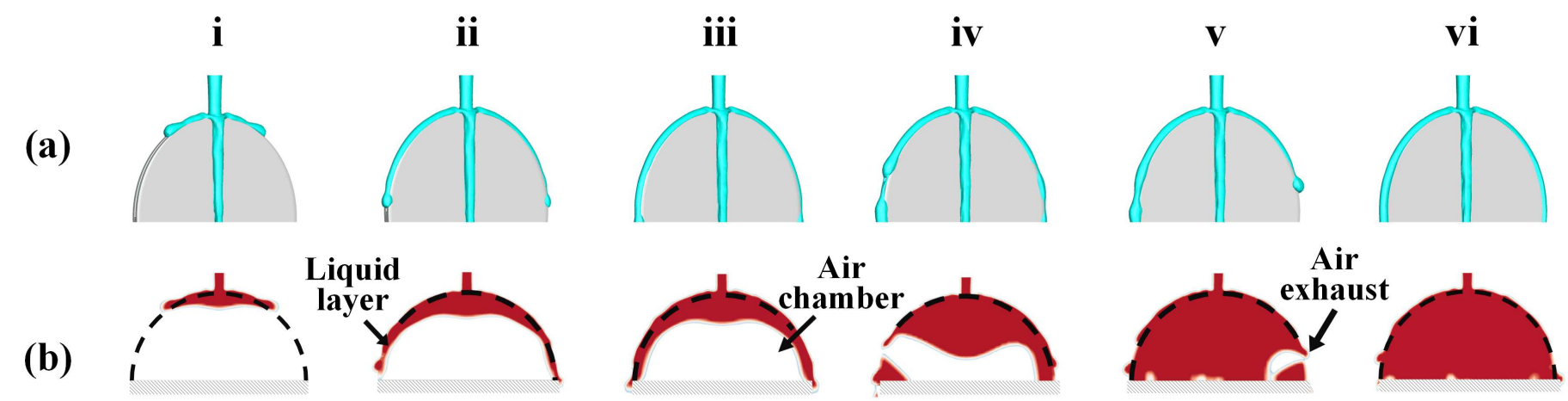

\section{$T(\mathbf{s}) \quad 0.14$}

0.31

0.45

1.86

3.74

4.08

Figure 8

Results of simulation with the kerf width of $0.1 \mathrm{~mm}$ : Please see manuscript .pdf for full caption 


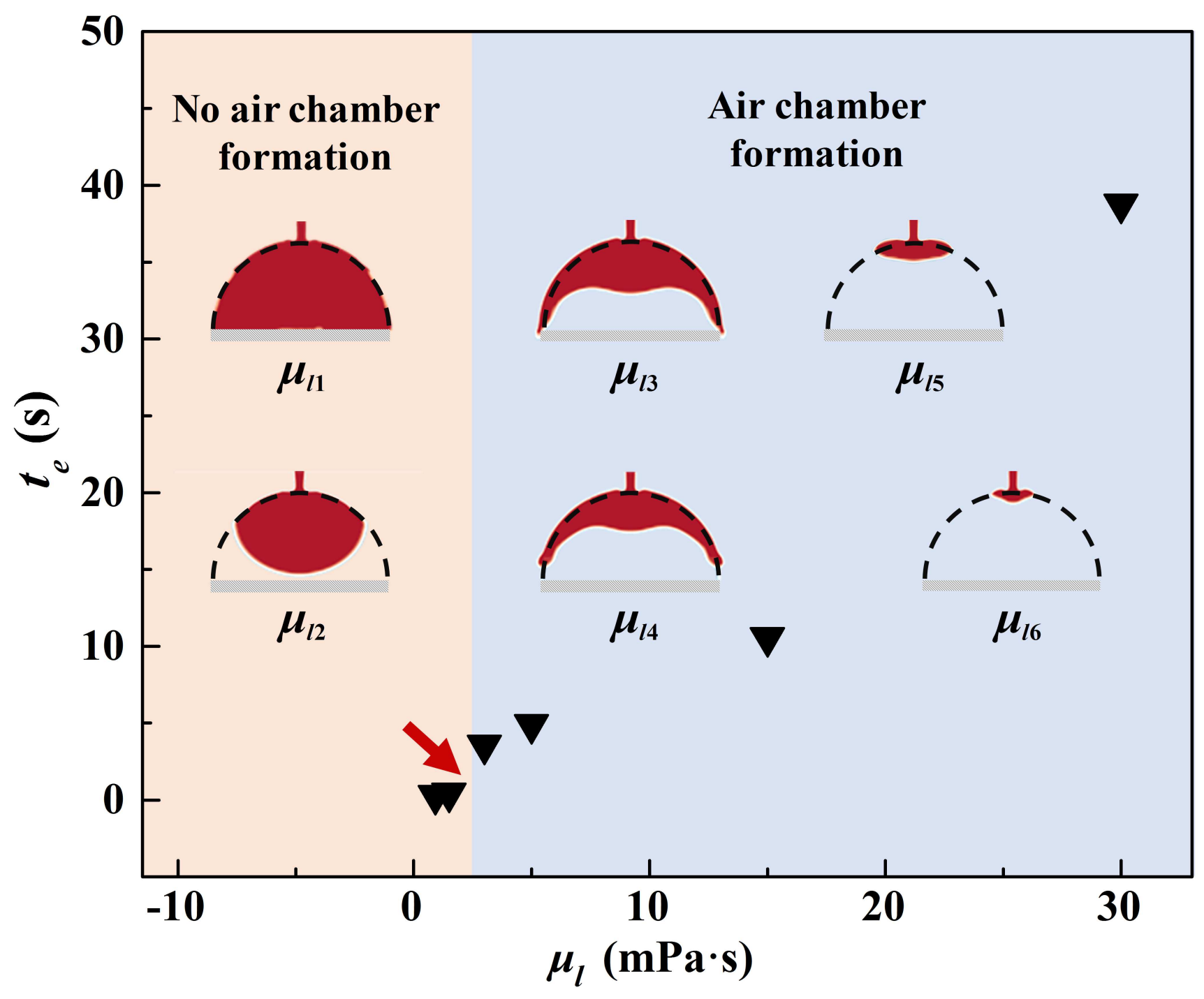

Figure 9

Plot of the liquid flow time to equilibrium with various liquid dynamic viscosity: Please see manuscript .pdf for full caption 


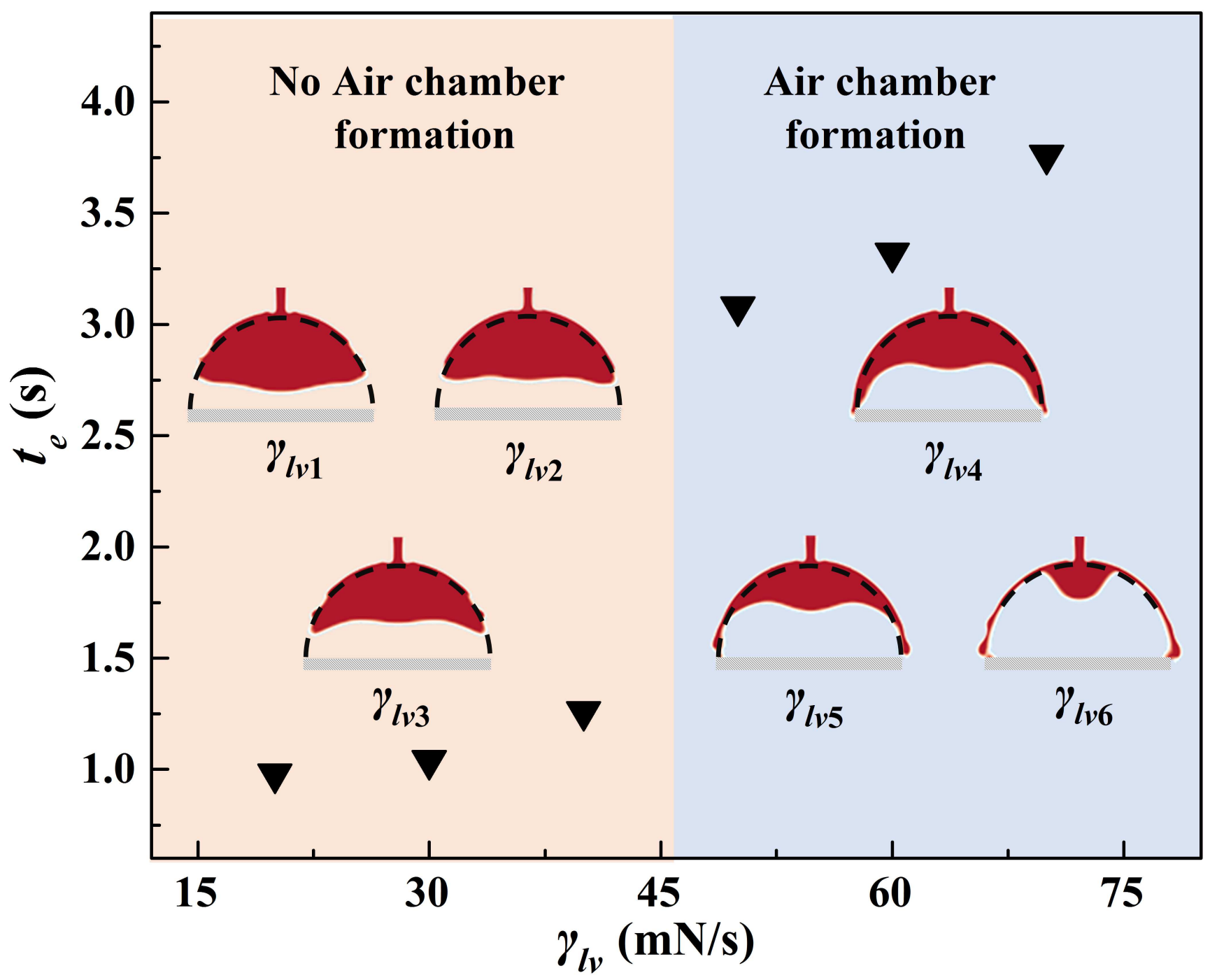

Figure 10

Plot of the time to the equilibrium state with various liquid surface tension: Please see manuscript .pdf for full caption 


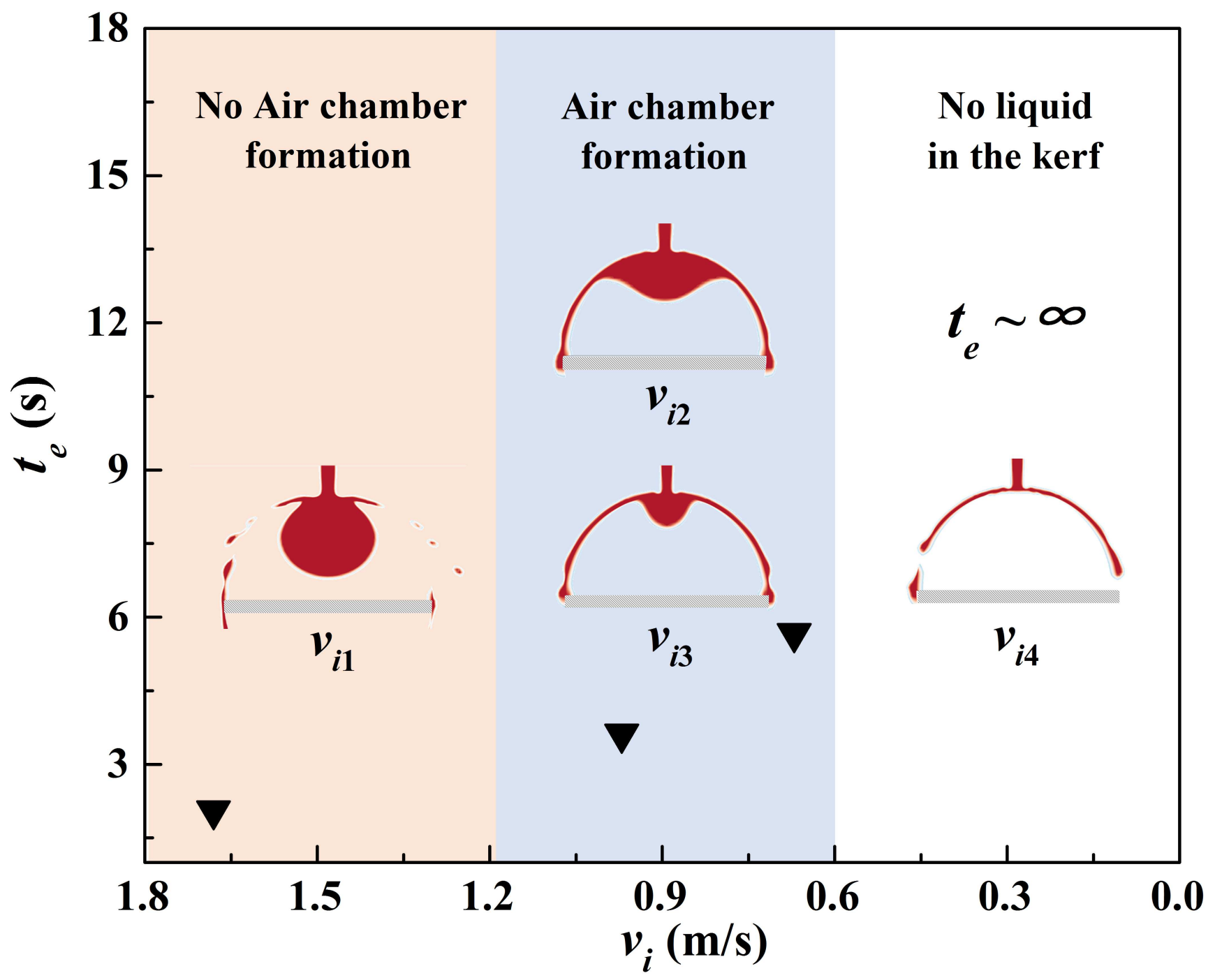

Figure 11

Plot of the time to the equilibrium state with various liquid velocity at the inlet area of the kerf: Please see manuscript .pdf for full caption 
(a)
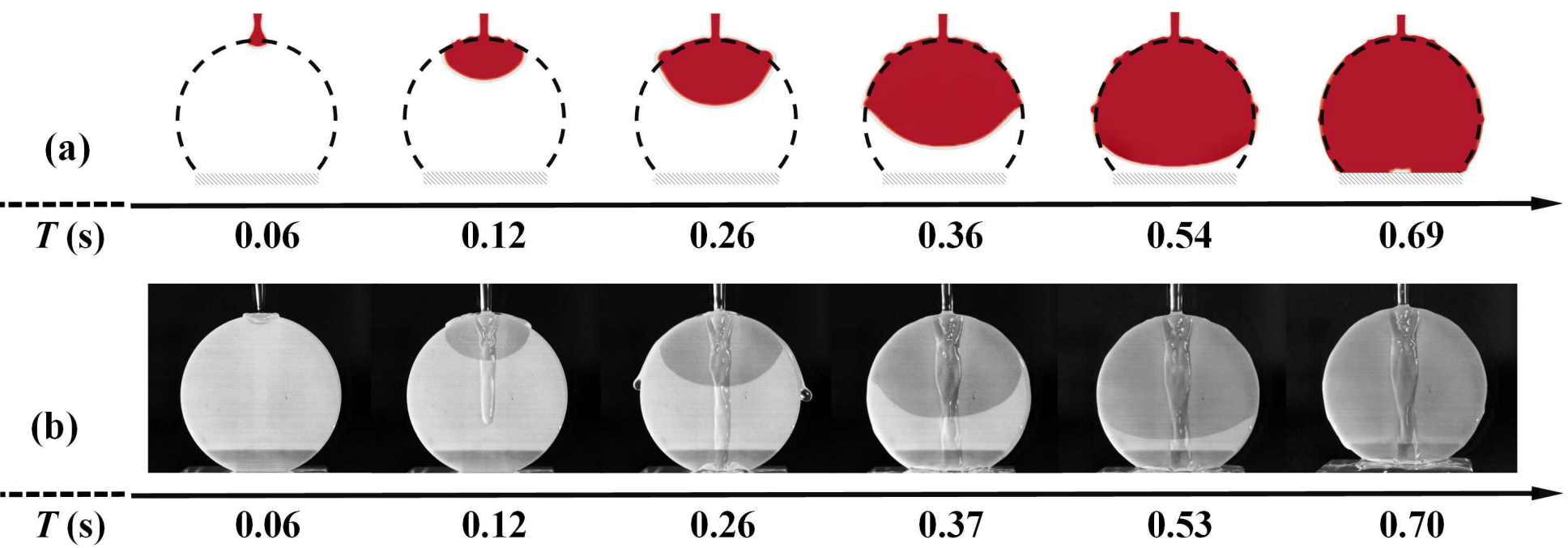

Figure 12

Transient evolution of the spreading contours of solution A in the kerf: Please see manuscript .pdf for full caption

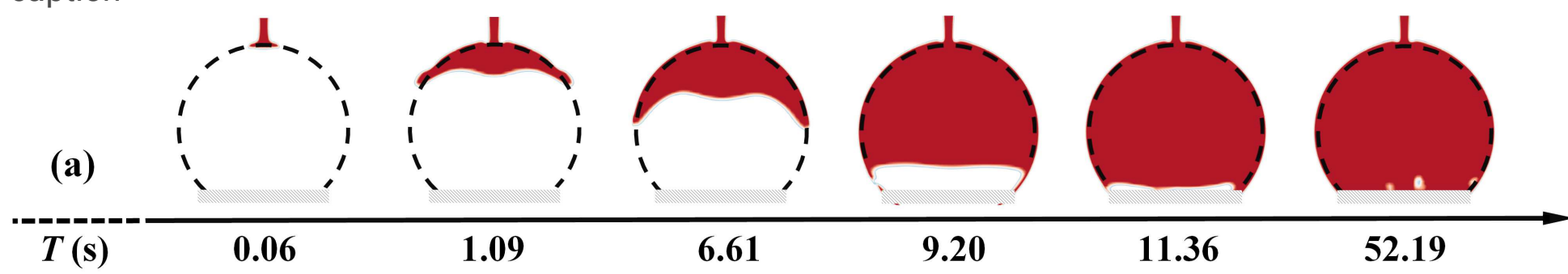

(b)

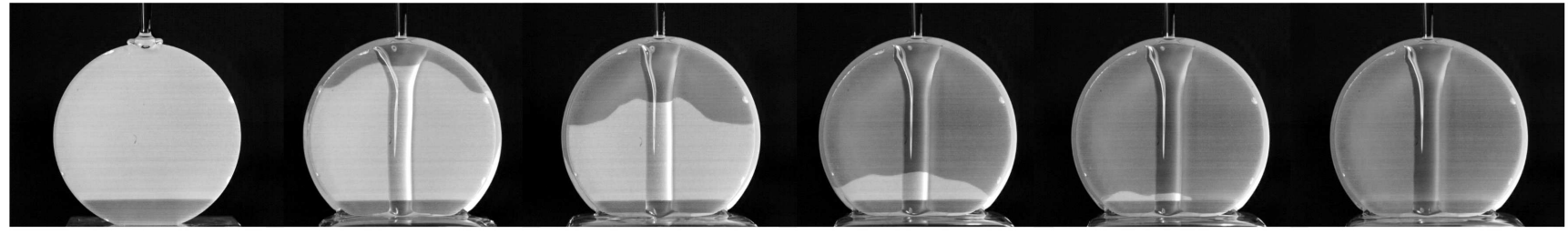

$\begin{array}{lllllll}T(\mathrm{~s}) & 0.06 & 1.14 & 6.70 & 9.24 & 11.48 & 52.31\end{array}$

Figure 13

Transient evolution of the spreading contours of solution B in the kerf: Please see manuscript .pdf for full caption 


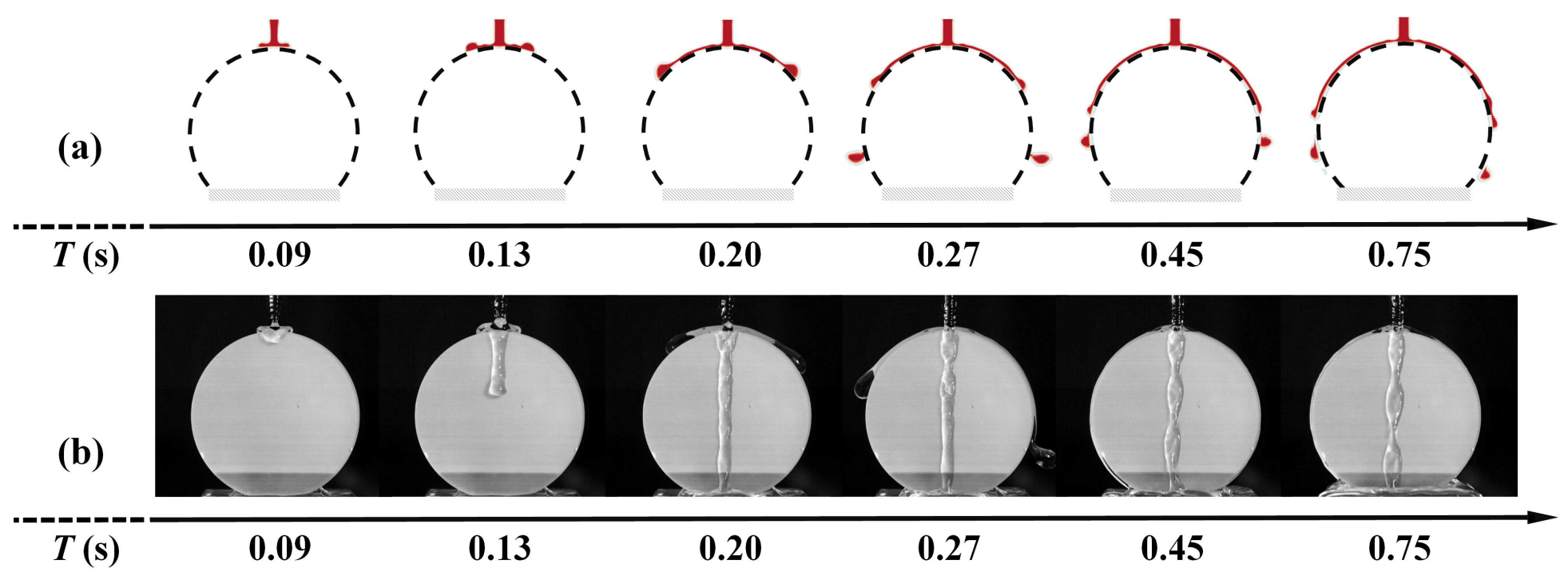

Figure 14

Transient evolution of the spreading contours of solution C in the kerf: Please see manuscript .pdf for full caption

(a)
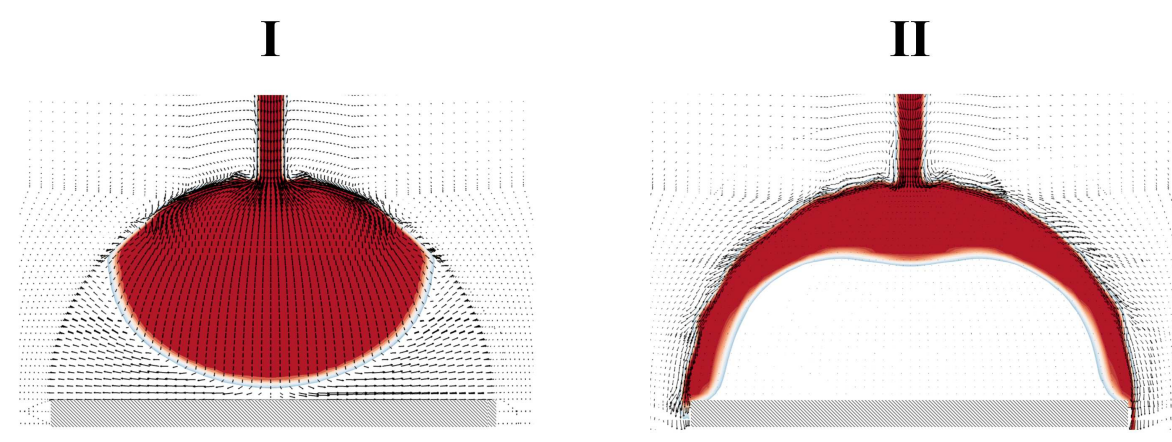

III

(b)
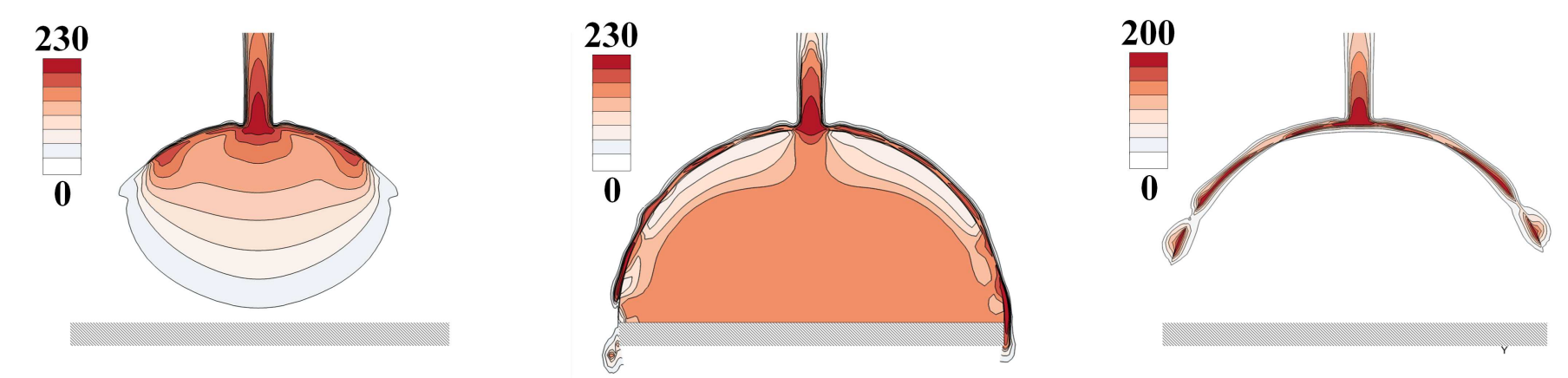

Figure 15

Three typical spreading regimes with corresponding (a) vector diagrams, (b) The total pressure nephograms. Please see manuscript .pdf for full caption 


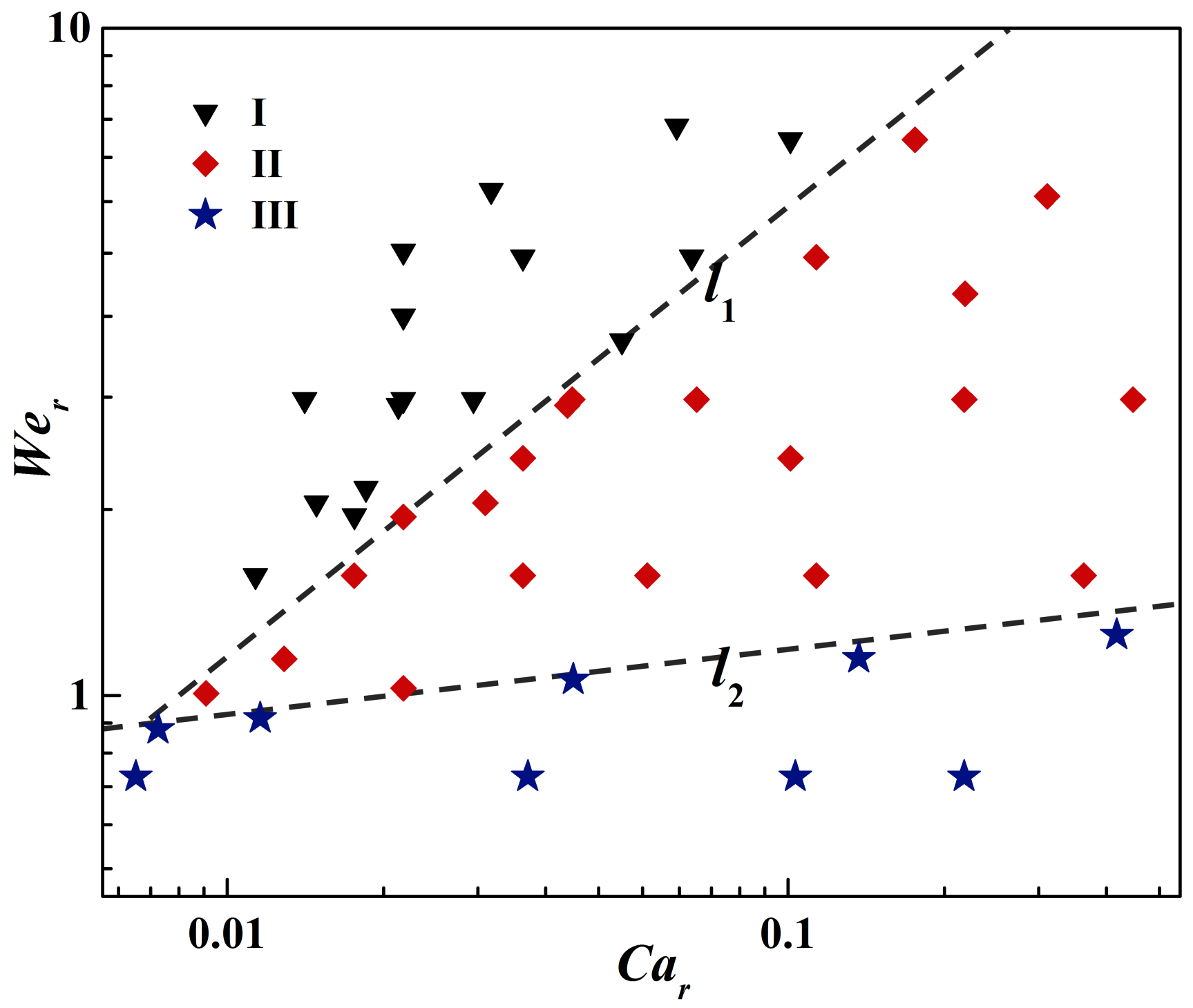

Figure 16

Non-dimensional map of the simulation results, Please see manuscript .pdf for full caption 